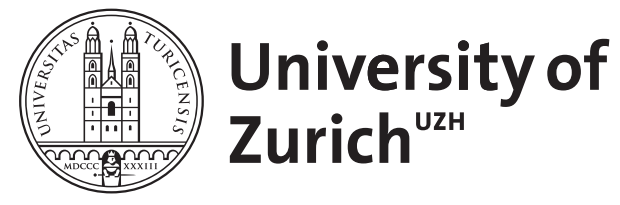

Zurich Open Repository and Archive

University of Zurich

University Library

Strickhofstrasse 39

CH-8057 Zurich

www.zora.uzh.ch

Year: 2011

Die strafrechtliche Bewältigung der Finanzkrise am Beispiel der Strafbarkeit wegen Untreue

Wohlers, Wolfgang

Posted at the Zurich Open Repository and Archive, University of Zurich

ZORA URL: https://doi.org/10.5167/uzh-58836

Journal Article

Published Version

Originally published at:

Wohlers, Wolfgang (2011). Die strafrechtliche Bewältigung der Finanzkrise am Beispiel der Strafbarkeit wegen Untreue. Zeitschrift für die gesamte Strafrechtswissenschaft (ZStW), 123:791-815. 


\title{
Die strafrechtliche Bewältigung der Finanzkrise am Beispiel der Strafbarkeit wegen Untreue*
}

\author{
Von Professor Dr. Wolfgang Wohlers, Zürich
}

\section{Die Finanzkrise als Anlass und Gegenstand der Zuweisung strafrecht- licher Verantwortlichkeit}

Großereignisse wie die durch den Zusammenbruch des Systems der Verbriefung forderungsbesicherter Wertpapiere (Asset Backed Securities) ausgelöste Finanzkrise $^{1}$ rufen nach Schuldigen ${ }^{2}$ : Die Öffentlichkeit und die Medien fordern die Zuweisung von Verantwortung, die Politik gibt dem Druck nach oder verstärkt diesen noch - entweder aus Überzeugung oder aber aus taktischen Gründen und auch die Strafjustiz steht unter Druck, ein Druck der zum Teil sicherlich von außen an die Staatsanwaltschaften und Gerichte herangetragen wird ${ }^{3}$, der aber wohl zumindest zu einem Teil auch hausgemacht ist: Auch die Strafbehörden wollen - aus sachlich begründetem Antrieb und/oder aus dem Bestreben heraus, sich der eigenen Bedeutung zu versichern - etwas „bewirken“. Dieses Bedürfnis hat aber nicht nur die Strafjustiz erreicht, sondern auch die Strafrechtswissenschaft. Auch die Strafrechtswissenschaft will nicht abseits stehen, sondern sie will einen Beitrag - wenn möglich: einen wichtigen Beitrag - zur Bewältigung der Krise leisten. Paradigmatisch hierfür ist eine Äußerung von Bernd Schünemann in einer Publikation mit dem vielleicht etwas reißerischen, aber jedenfalls prägnanten Titel „Die sogenannte Finanzkrise - Systemversagen oder global organisierte Kriminalität“. Nach Scbünemann geht es darum zu verhindern, dass später

* Um Fußnoten ergänzte und an einigen Stellen auch inhaltlich leicht erweiterte Fassung des Manuskripts, das dem Impulsreferat zugrunde lag, das der Verfasser am 26.6.2011 auf der 34. Strafrechtslehrertagung in Leipzig gehalten hat. Für eine kritische Durchsicht der ersten Fassung des Manuskripts dankt der Verfasser Frau Dr. Gunbild Godenzi.

1 Zur Entstehung und zum faktischen Ablauf der Finanzkrise vgl. im Einzelnen Schröder, Handbuch Kapitalmarktstrafrecht, 2. Aufl. 2010, Rdn. 1082 ff.; ders., Kriminalistik 2009, 12 ff.; ders., NJW 2010, 1169 ff.; Brüning/Samson, ZIP 2009, 1089 f.; Florstedt, AG 2010, 315 f.; Gallandi, wistra 2009, 41 ff.; Gillmeister, in: Kempf/Lüderssen/Volk (Hrsg.), Die Finanzkrise, das Wirtschaftsstrafrecht und die Moral, 2010, S. 280 ff.; Hellwig, Finanzmarktregulierung Welche Regelungen empfehlen sich für den deutschen und europäischen Finanzsektor?, Finanzkrise und Reformbedarf, Gutachten E zum 68. Deutschen Juristentag, in: Verhandlungen des 68. Deutschen Juristentages Berlin 2010, Band I, 2010, E 12 ff., 24 ff.; Kasiske, in: Schünemann (Hrsg.), Die sogenannte Finanzkrise - Systemversagen oder global organisierte Kriminalität?, 2010, S. 14 ff.; Lüderssen, StV 2009, 486, 489; Lutter, ZIP 2009, 197 f.; Otto, Festschrift für für Krey, 2010, S. 379 ff.; Park/Rütters, StV 2011, 434 ff.; Ransiek, WM 2010, 869 f.; Rudolph, ZGR 2010, 1, 4 ff.; Schünemann, in: Schünemann (Hrsg.), a. a. O., S. $74 \mathrm{ff.}$

2 Deiters, in: Kempf/Lüderssen/Volk (Anm.1), S.132; Trüg, in: Kempf/Lüderssen/Volk (Anm. 1), S. 267 f.; Hank, in: Kempf/Lüderssen/Volk (Anm. 1), S. $363 \mathrm{ff.}$

3 Vgl. Jean-Richard-dit-Bressel, schwZStrR 126 (2008), S. 115, 116, der die Situation aus seiner Sicht eines Züricher Staatsanwalts wie folgt umschreibt: „Auf ihnen [den Strafbehörden] lastet die Erwartung, ,den wahren Schuldigen' zur Strecke zu bringen“. 
gesagt werde, „die deutsche Strafrechtswissenschaft habe sich wieder einmal in eine Nische zurückgezogen, als es ernst wurde“ ${ }^{4}$.

Die skizzierten Mechanismen greifen - wenn auch in abgeschwächter Form sogar dann, wenn es um die „Bewältigung“ von Naturereignissen geht. Als Hamburger denkt man an Flutkatastrophen, als in der Schweiz wohnhafter Hamburger an Bergrutsche und Lawinen. Die Suche nach Schuldigen hat aber vor allem dann Konjunktur, wenn es um Katastrophen geht, die von Menschenhand gemacht worden sind, wie z.B. Entscheidungen von Managern und anderen Entscheidungsträgern im Wirtschaftsleben, die sich - jedenfalls nachträglich betrachtet - als Fehlentscheide darstellen ${ }^{5}$. Und damit sind wir dann bei der Finanzkrise angekommen, die entgegen einer gelegentlich geäußerten These kein Ereignis ist, das quasi wie eine Naturgewalt über uns gekommen ist. Wir haben es nicht mit einem autonom ablaufenden reinen Systemversagen zu tun, sondern wir haben es mit einem Ereignis zu tun, das von Menschenhand gemacht worden ist $^{6}$ und für dessen Entstehen und Entwicklung damit dann auch das Bestehen personaler Verantwortlichkeiten nicht von vornherein von der Hand gewiesen werden kann?.

Es wäre nun aber kurzschlüssig, wenn man aus diesem Befund folgern würde, dass die Finanzkrise zwingend auch strafrechtliche Verantwortlichkeit nach sich ziehen muss. Dass dieser Schluss unstatthaft ist, zeigen bereits sehr viel überschaubarere Krisen, wie z.B. die Krise, die ich kurz nach meinem Wechsel nach Zürich sozusagen vor Ort mitverfolgen konnte: das Grounding der Swissair am 2. Oktober 2001. Die strafrechtliche Aufarbeitung dieses Unternehmenskonkurses, die von der Zürcher Staatsanwaltschaft mit erheblichem personellen und finanziellen Aufwand betrieben worden war ${ }^{8}$, ist letztlich ausgegangen wie das sprichwörtliche Hornberger Schießen: Das Verfahren vor dem Bezirksgericht Bülach endete mit Freisprüchen für alle 19 Angeklagten ${ }^{9}$, die - soweit angefochten - durch das Obergericht Zürich bestätigt worden sind ${ }^{10}$. Dass niemand für

4 Schünemann, in: Schünemann (Anm. 1), S. 5.

5 Beulke, Festschrift für Eisenberg, 2009, S. 266: Die Suche nach einem Schuldigen habe alle Lebensbereiche erreicht und mache auch vor dem Wirtschaftssektor nicht halt.

6 Wandt, in: Kempf/Lüderssen/Volk (Anm. 1), S. 3; Vaubel, in: Kempf/Lüderssen/Volk (Anm. 1), S. 22; zu den Gründen, die dazu geführt haben, dass die einzelnen Akteure so agiert haben, wie sie es getan haben, vgl. Hellwig (Anm. 1), E 35 ff.; Spindler, AG 2010, S. 601, 602 f.; Zimmer, Finanzmarktregulierung - Welche Regelungen empfehlen sich für den deutschen und den europäischen Finanzsektor?, Gutachten G zum 68. Deutschen Juristentag, 2010, G $14 \mathrm{ff}$.

7 Bittmann, NStZ 2011, 361, 361/362; Kasiske, in: Schünemann (Anm. 1), S. 37 f.; Strate, HRRS 2009, 441; skeptisch im Hinblick auf die Möglichkeit, strafrechtliche Verantwortlichkeit zuzusprechen: Jahn, JZ 2011, 340, 345; Vaubel, in: Kempf/Lüderssen/Volk (Anm. 1), S. 24; vgl. auch Lüderssen, $\mathrm{StV} 2009,486 \mathrm{ff}$.

8 Die Ermittlungsakten der Staatsanwaltschaft sollen dem Vernehmen nach 4000 Aktenordner umfasst haben, was für die Strafverfolgungsbehörden der Schweiz jedenfalls quantitativ gesehen eine neue Dimension eröffnet hat.

9 Urteile des Bezirksgerichts Zürich vom 4.6.2007 und hierzu die Darstellung und Würdigung bei Schubarth/Peter, SZW 2008, 455.

10 Vgl. Urteil des Obergerichts Zürich vom 20.6.2008 in Sachen Mario Corti (vgl. http:// 
den Untergang der Swissair strafrechtlich verantwortlich sein soll, ist für die Öffentlichkeit, die Medien und wohl auch für die Politik in der Schweiz nur schwer zu akzeptieren gewesen ${ }^{11}$. Wenn man aber akzeptiert und anerkennt, dass auch traumatisierende Ereignisse nicht allein deshalb strafbar sein können, weil sie traumatisierend sind, sondern dass sie nur dann strafbar sind, wenn die im geltenden Recht geregelten Straftatbestände erfüllt sind, dann war dieses Ergebnis eigentlich mehr oder weniger absehbar: Das ursprüngliche Management, das die Entscheide getroffen hat, die sich jetzt - rückblickend - als Fehlentscheide darstellen, kann sich darauf berufen, dass man zum damaligen Zeitpunkt berechtigterweise davon ausgehen durfte, dass es sich nicht um Fehlentscheide gehandelt hat; und das in der Krise agierende Management konnte sich darauf berufen, dass es eigentlich selbst nicht mehr viel falsch gemacht hat, sondern die Suppe auszulöffeln hatte, die ihm durch die Entscheidungen des vormaligen Managements eingebrockt worden ist.

Das geschilderte Szenario ist nun weder ein Sonderfall Swissair noch ein Sonderfall Schweiz, sondern es ist ein Muster, auf das man gerade in Wirtschaftsstrafsachen immer wieder stößt. Und vor diesem Hintergrund stellt sich dann natürlich in besonderer Dringlichkeit die Frage, ob es nicht vielleicht sogar etwas größenwahnsinnig ist, Katastrophen vom Kaliber der Finanzkrise mit den Mitteln des Strafrechts „bewältigen“ zu wollen. Wenn man sich einer Antwort auf diese Frage annähern will, dann muss allerdings zunächst einmal klargestellt werden, was man eigentlich meint, wenn man von „Bewältigung“ der Finanzkrise redet.

\section{Bewältigung der Finanzkrise als präventive Vermeidung zukünftiger Finanzkrisen}

Üblicherweise - etwa im Zusammenhang mit den beim modernen Gesetzgeber so überaus beliebten „Bekämpfungsgesetzen“12 - wird mit Bewältigung wohl die

www.nzz.ch/nachrichten/wirtschaft/aktuell/freispruch_fuer_mario_corti_rechtskraeftig 1.965696.html; abgerufen am 19.12.2011).

11 Vgl. hierzu auch das auf die Notwendigkeit der strafrechtlichen Aufarbeitung bezogene zusammenfassende Fazit bei Schubarth/Peter, SZW 2008, 455, 464: „Das Grounding der Swissair war die schlimmste Niederlage der Eidgenossenschaft seit der Schlacht von Marignano. Der Swissair-Prozess war deshalb möglicherweise aus Gründen der Volkstherapie eine Notwendigkeit, zumindest in der Form von Balsam auf die Seele einer zutiefst verletzten Nation." Dass diese Analyse wohl zutreffend ist, belegt der Umstand, dass der Konkurs der Swissair neben mehreren Büchern (vgl. z. B. Moser, Bruchlandung: wie die Swissair zugrunde gerichtet wurde, Zürich 2001; Lüchinger, Der Fall der Swissair: das Drama, der Untergang, die Akteure, 2. Aufl., Zürich 2001; ders. [Hrsg.], Swissair: Mythos \& Grounding, Zürich 2006) auch in einem als „Doku-Thriller“ vermarkteten Film (Grounding - Die letzten Tage der Swissair, 2006) „verarbeitet“ wurde.

12 Vgl. Hettinger, NJW 1996, 2263, 2264; Woblers, Deliktstypen des Präventionsstrafrechts - zur Dogmatik „moderner“ Gefährdungsdelikte, 2000, S.36ff. sowie - speziell zu den Gesetzen zur Bekämpfung der Wirtschaftskriminalität - S. $148 \mathrm{ff}$. 
Beseitigung bestehender und die präventive Verhinderung zukünftiger Krisen gemeint. Wenn man den Begriff so versteht, dann muss man aber wohl wirklich skeptisch sein: Strafrecht ist, dies dürfte die Diskussion um das „moderne“ (Präventions-)Strafrecht ${ }^{13}$ in aller Deutlichkeit erwiesen haben, ein ungeeignetes Instrument, um soziale Krisen zu bewältigen. Ich beschränke mich an dieser Stelle darauf, beispielhaft auf den mit großem Elan und großen Erwartungen gestarteten und dann doch grandios gescheiterten, mit den Mitteln des Strafrechts geführten „War on Drugs“ 14 zu verweisen: Unabhängig davon, ob man es für notwendig und/oder legitim erachtet, auf den Konsum bestimmter, als illegal definierter Substanzen mit den Mitteln des Strafrechts zu reagieren ${ }^{15}$, wird man anerkennen müssen, dass das Strafrecht nicht in der Lage ist, das im Konsum dieser Substanzen liegende Problem zu bewältigen ${ }^{16}$.

Dass die Steuerungsfähigkeit des Strafrechts bezogen auf das Wirtschaftsleben signifikant größer ist, wird man nicht annehmen können ${ }^{17}$. Strafrecht kann nur über Abschreckung bzw. Norminternalisierung wirken und diese Wirkungen sind nach allem, was wir wissen, sehr beschränkt ${ }^{18}$. Strafrecht ist im Übrigen aber auch eine sekundäre Normenordnung, die an die Wertungen der Primärrechtsordnung anknüpfen muss, wobei aus dieser Angewiesenheit auf die (Vor-)Wertungen der Primärrechtsordnung ${ }^{19}$ folgt, dass die auf die Bewältigung und/oder Vermeidung von krisenhaften Situationen abzielenden Maßnahmen mit den Instrumenten der Primärrechtsordnung erreicht oder doch zumindest

Grundlegend hierzu Prittwitz, Strafrecht und Risiko, 1993; zur Auseinandersetzung mit den insbesondere von Frankfurter Kollegen vertretenen Ansätzen vgl. auch Wohlers (Anm. 12), S. $49 \mathrm{ff}$.

14 Zur Entwicklung des Betäubungsmittelstrafrechts in Deutschland und in der Schweiz vgl. Woblers (Anm. 12), S. $178 \mathrm{ff}$. sowie - auch zur Entwicklung in den Niederlanden - Woblers/ Went, in: von Hirsch/Neumann/Seelmann (Hrsg.), Paternalismus im Strafrecht, 2010, S. 299 ff.

15 Zur zweifelhaften Legitimität des geltenden Betäubungsmittelstrafrechts vgl. P. Albrecht, Die Strafbestimmungen des Betäubungsmittelgesetzes (Art.19-28 BetmG), 2. Aufl., Bern 2007, Einl. Rdn. 36 ff.; Haffke, ZStW 107 (1995), S.761; Köhler, ZStW 104 (1992), S. 3; Nestler, in: Kreuzer (Hrsg.), Handbuch des Betäubungsmittelstrafrechts, 1998, $\$ 11$; Woblers (Anm. 12), S. $190 \mathrm{ff} ., 340 \mathrm{f}$.

16 Vgl. Malek, Betäubungsmittelstrafrecht, 3. Aufl. 2008, Einl. Rdn. 12 ff., insbes. Rdn. 24.

17 Zur Skepsis gegenüber dem Steuerungspotenzial des Wirtschaftsstrafrechts vgl. Hefendehl, ZStW 119 (2007), S. 816, 820 ff.; Lüderssen, StV 2009, 486, 493 f.; ders., in: Kempf/Lüderssen/ Volk (Hrsg.), Die Handlungsfreiheit des Unternehmers - wirtschaftliche Perspektiven, strafrechtliche und ethische Schranken, 2009, S. 284 ff.; Ransiek/Hüls, ZGR 2009, 157, 182; vgl. aber auch - tendenziell optimistischer - Schmidt, in: Kempf/Lüderssen/Volk a. a. O., S. $101 \mathrm{f}$.

18 Vgl. Dölling, ZStW 102 (1990), S. 1, 5 ff.; Frischknecht, Kultureller Rabatt, Bern 2009, S. 132 ff., 143 ff.; Meier, Strafrechtliche Sanktionen, 2. Aufl. 2009, S. 27 ff.; Müller-Tuckfeld, Integrationsprävention, 1998, S. 100 ff., 115 ff.; Schöch, Festschrift für Jescheck, 1985, Zweiter Halbband, S. 1098 ff.; Streng, Strafrechtliche Sanktionen, 2.Aufl. 2002, Rdn. 54 ff.; Woblers (Anm. 12), S. $57 \mathrm{ff}$.

19 Der Charakter des Strafrechts als sekundäre Normenordnung darf nicht mit der Diskussion um die Subsidiarität des Strafrechts gegenüber dem Zivil- und sonstigen Öffentlichen Recht vermengt oder gar verwechselt werden; zur Diskussion um die Subsidiarität des Strafrechts vgl. unten IV. 
vorgezeichnet werden müssen ${ }^{20}$. Die Funktion des Strafrechts kann allein darin liegen, die durch die Primärrechtsordnung gesetzten Regelungen abzusichern ${ }^{21}$.

Zutreffend hat Achenbach in seinem Referat auf der Strafrechtslehrertagung in Osnabrück festgehalten, dass Strafrecht eine „sekundäre Normenordnung“ sei und dass speziell das Wirtschaftsstrafrecht die Funktion habe, Maßnahmen flankierend abzusichern, „die ausserhalb seiner [des Strafrechts] Grenzen getroffen und verantwortet werden"22. Hieraus folgt dann aber - worauf Ransiek im Zusammenhang mit der aktuellen Finanzkrise hingewiesen hat -, dass es „von vornherein aussichtslos [ist], strukturelle Probleme, die Folge des vernetzten Verhaltens einer Vielzahl von Akteuren weltweit [sind], mit den Mitteln des Strafrechts adäquat erfassen zu wollen“"23. Und speziell bezogen auf den Untreuestraftatbestand hat Saliger schon vor mehr als 10 Jahren „die grundsätzliche Untauglichkeit der (allgemeinen) Untreuevorschrift zur Lösung von spezifischen Ordnungsproblemen des Zivilrechts und des öffentlichen Rechts“ postuliert ${ }^{24}$.

Aus alledem wird man den Schluss ziehen müssen, dass dann, wenn es um Bewältigung im Sinne von zukunftsorientierter Vermeidung zukünftiger Finanzkrisen geht, mit den Mitteln des Finanz- und Kapitalmarktrechts gearbeitet werden muss ${ }^{25}$ und dass folgerichtig die Rolle des weißen Ritters nicht der Strafrechtswissenschaft zukommt, sondern wohl eher den Finanz- und Kapitalmarktrechtlern, während wir Strafrechtler allenfalls in der Rolle des Knappen mitspielen. Bezogen auf die aktuelle Finanzkrise kommt dann noch hinzu, dass es sich um eine internationale Krise handelt, bei der auch nationale Gesetzgeber für sich allein praktisch nichts ausrichten können. Wenn man sich den durch verschiedene internationale Tagungen zwischenzeitlich ja schon recht gut aufgearbeiteten Flickenteppich der weltweit existenten nationalen Untreueregelungen ${ }^{26}$ ansieht, dann wird schnell klar, dass das nationale Untreuestrafrecht - sei es nun das deutsche, das österreichische, das schweizerische oder sei es das Strafrecht irgendeines anderen Nationalstaats - ein von vornherein ungeeignetes Instrument ist, wenn es darum geht, die Welt vor derartigen global ablaufenden Ereignissen zu bewahren.

\footnotetext{
Lüderssen, in: Kempf/Lüderssen/Volk (Anm.1), S. $15 \mathrm{ff.}$

Nestler, in: Kempf/Lüderssen/Volk (Anm.17), S. $91 \mathrm{f}$.

Achenbach, ZStW 119 (2007), S. 789.

Ransiek, WM 2010, 869.

Saliger, ZStW 112 (2000), S. 563, 564.

$25 \mathrm{Zu}$ den diesbezüglichen Vorschlägen vgl. Hellwig (Anm. 1), E 49 ff.; Otto, WM 2010, 2013, 2014 ff.; Rudolph, ZGR 2010, 1, 26 ff.; Spindler, AG 2010, 601, 603 ff.; Wittig, WM 2010, 2337 ff.; Zimmer (Anm. 5), G 25 ff. ; vgl. auch Otto, Festschrift für Krey, 2010, S. 396 ff.

26 Vgl. hierzu die Beiträge von Foffani und Zerbes, in: Kempf/Lüderssen/Volk (Anm. 1), S. $141 \mathrm{ff}$. und $158 \mathrm{ff}$. sowie die Beiträge von Rönnau, du Bois-Pedain, Luzón Peña/Roso Cañadillas und Foffani, ZStW 122 (2010), 299 ff.; vgl. auch Cappel, Grenzen auf dem Weg zu einem europäischen Untreuestrafrecht, 2009, S. $187 \mathrm{ff}$.
} 


\section{Die Bewältigung der Finanzkrise als Synonym für die - auch strafrecht- liche - Aufarbeitung der Finanzkrise}

Wenn die Diskussion über die strafrechtliche Bewältigung der Finanzkrise Sinn machen soll, dann muss mit „Bewältigung“ etwas anderes als zukunftsorientierte Vermeidung zukünftiger Finanzkrisen gemeint sein. Man muss dann Bewältigung retrospektiv, d.h. im Sinne von Aufarbeitung der geschehenen Krise verstehen. Der - für sich gesehen zutreffende - Einwand, dass Strafrecht ein ungeeignetes und untaugliches Mittel ist, wenn es darum geht, historische Ereignisse aufzuarbeiten ${ }^{27}$, steht diesem Ansatz nicht entgegen: Auch wenn eine Krise - wie die Finanzkrise - als Ganzes nicht einer oder mehreren Personen zur Last gelegt werden kann, steht doch andererseits auch außer Frage, dass die einzelnen, auf dem Finanzmarkt agierenden Akteure für ihr je individuelles Verhalten zur Verantwortung gezogen werden können und im Hinblick auf das Legalitätsprinzip auch zur Verantwortung gezogen werden müssen, wenn und soweit sie mit ihrem Verhalten Straftatbestände erfüllt haben.

Es geht hierbei natürlich nicht darum, eine oder mehrere Person für die Finanzkrise als solche zur Verantwortung zu ziehen. Dies schon deshalb nicht, weil es de lege lata gar keine Straftatbestände gibt, die die Krise als solche erfassen. Es geht immer nur um einzelne Teilbeiträge und auch diese werden nicht einmal wegen ihres Charakters als Teil der Krise erfasst, sondern aus anderen Gründen, z. B. weil sie Schäden bei anderen Marktteilnehmern oder beim eigenen Unternehmen verursacht haben. Wenn wir von strafrechtlicher Bewältigung der Finanzkrise sprechen, geht es also schlicht und einfach darum, ob bestimmte Personen durch ihr Verhalten im Vorfeld oder im Rahmen der Krise konkrete Straftatbestände erfüllt haben ${ }^{28}$.

Wenn andere Marktteilnehmer geschädigt wurden, steht insoweit der Straftatbestand des Betrugs im Vordergrund ${ }^{29}$, wobei dann entscheidend ist, ob eine betrugsrelevante Täuschung vorliegt oder aber der Geschädigte sehenden Auges ein Risiko eingegangen ist. Während man letzteres im Verhältnis der Banken untereinander wohl eher annehmen kann oder sogar annehmen muss ${ }^{30}$, kann im Verhältnis der Finanzmarktdienstleister zu ihren in Finanzgeschäften nicht

27 Vgl. Deiters, in: Kempf/Lüderssen/Volk (Anm. 1), S. 132.

28 Vgl. auch bereits Ransiek, WM 2010, 869 sowie Fischer, in: Kempf/Lüderssen/Volk (Anm. 1), S. 196 f. mit dem zutreffenden Hinweis darauf, dass auch dann, wenn das Strafrecht soziale Probleme nicht lösen könne, individuelles Fehlverhalten trotzdem geahndet werden müsse.

29 Vgl. auch Bittmann, NStZ 2011, 361, 362; Park/Rütters, StV 2011, 434, 440 f.; Schünemann, in: Schünemann (Anm. 1), S. 81.

30 Vgl. Gallandi, wistra 2009, 41, 43 f.; Ransiek, WM 2010, 869, 873. In besonderer Weise gilt dies für die schweizerische Rechtsordnung, da nach Art. 146 schwStGB nicht jede Täuschung ausreicht, sondern es sich um eine arglistige Täuschung handeln muss; vgl. hierzu Stratenwerth/Jenny/Bommer, Strafrecht Besonderer Teil I: Straftaten gegen Individualinteressen, 7. Aufl., Bern 2010, \$15 Rdn. 17 ff.; Stratenwerth/Woblers, Schweizerisches Strafgesetzbuch, Handkommentar, 2. Aufl., Bern 2009, Art. 146 Rdn. 6. 
bewanderten Kunden durchaus etwas anderes gelten ${ }^{31}$. Dass insoweit Potenzial für die Intensivierung der Zuweisung strafrechtlicher Verantwortlichkeit vorhanden ist, belegt eine Entscheidung des Bundesgerichtshofs in Zivilsachen, mit der vor einigen Wochen bezogen auf den Verkauf von Finanzprodukten durch Banken ein recht deutliches Zeichen gesetzt wurde, als der BGH eine Verletzung der Aufklärungspflicht bezogen auf ein komplexes Anlageprodukt (CMS Spread Ladder Swap) bejaht hat ${ }^{32}$.

Wenn es nicht um die Schädigung von Kunden oder anderen Marktteilnehmern geht, sondern um Schädigungen des eigenen Unternehmens bzw. der Anteilseigner dieses Unternehmens, dann steht nicht die Strafbarkeit wegen Betruges im Vordergrund ${ }^{33}$, sondern diejenige wegen Untreue ${ }^{34}$, womit wir dann bei der zentralen Frage dieses Referats angelangt sind: Was kann das Untreuestrafrecht im Rahmen der Aufarbeitung des Verhaltens leisten, das zur aktuellen Finanzkrise geführt hat? Bevor wir uns dieser Frage zuwenden, ist allerdings zunächst noch auf einen Einwand einzugehen, den man vor die Klammer ziehen kann und vielleicht sogar vor die Klammer ziehen muss. Dieser Einwand betrifft den mit dem Schlagwort des Subsidiaritätsprinzips verbundenen Charakter des Strafrechts als Ultima Ratio.

\section{Der Einwand der Subsidiarität des Einsatzes strafrechtlichen Zwangs}

Der gerade im Hinblick auf das Wirtschaftsstrafrecht ${ }^{35}$ immer wieder erhobene Einwand geht dahin, dass Strafrecht erst und nur dann zur Anwendung kommen dürfe, wenn andere Möglichkeiten der Reaktion nicht gegeben $\operatorname{sind}^{36}$. Dieser Einwand ist nach hier vertretener Auffassung im Ergebnis nicht überzeugend:

Der gerade im Zusammenhang mit dem Wirtschaftsstrafrecht immer wieder gern vorgebrachte Verweis auf die Selbstheilungskräfte des Marktes sollte sich mittlerweile wohl endgültig als lebensfremde Annahme erwiesen haben: „Den hier offenbar gewordenen Missständen ist auch nicht mehr mit freiwilligen Übereinkommen, moralischen Appellen oder Besinnung auf moralische Werte und Verpflichtungen beizukommen. Hier ist der Gesetzgeber gefordert “37.

Was damit als alternative Handlungsoption verbleibt ist das Aufsichtsrecht

31 Vgl. Ransiek, WM 2010, 869, 873; Rönnau, in: Schünemann (Anm. 1), S. 46 ff.; Schünemann, in: Schünemann (Anm. 1), S. $82 \mathrm{ff}$.

32 Vgl. BGH, Urteil vom 22.3.2011 - XI ZR 33/10.

33 Diese kommt wohl nur in den Fällen der Täuschung von Entscheidungsträgern durch andere Mitarbeiter in Betracht.

34 Schünemann, in: Schünemann (Anm. 1), S. 81.

35 Vgl. Haffke, KritV 1991, 165 ff. sowie - speziell zum Untreuestraftatbestand - Dierlamm, StraFo 2005, 397, 398.

$36 \mathrm{Zu}$ den insoweit zur Verfügung stehenden alternativen Instrumenten vgl. den Überblick bei Lüderssen, in: Kempf/Lüderssen/Volk (Anm. 17), S. $301 \mathrm{ff.}$

37 Vgl. Otto, Festschrift für Krey, 2010, S. 398. 
und das Zivilrecht, wobei das in jüngster Zeit zunehmend beliebte Instrument der staatlich gelenkten Selbstregulierung als das wahrgenommen werden sollte, was es wirklich ist: ein für den Staat kostengünstiger Ansatz des Outsourcing von Strafverfolgungsaufgaben ${ }^{38}$. Im Ergebnis geht dann allerdings auch der Verweis auf das Aufsichts- und/oder Zivilrecht ins Leere: Auch wenn man nicht so weit geht, dass man den Einsatz strafrechtlicher Instrumente mit Tiedemann als das gegenüber dem Wirtschaftsaufsichtsrecht mildere Mittel einstuft ${ }^{39}$, ist doch zunächst einmal darauf zu verweisen, dass das Strafrecht im geltenden Recht regelmäßig als Instrument eingesetzt wird, mit dem die Durchsetzung der Instrumente der Primärrechtsordnung abgesichert werden (muss) ${ }^{40}$. Hinzu kommt, dass auch das beste Aufsichts- und/oder Haftungsrecht nicht ausschließen kann, dass zumindest in einzelnen Fällen gegen die dortigen Regeln verstoßen wird, womit sich dann die Frage stellt, wie auf derartige Ausreißer reagiert werden soll. Jedenfalls dann, wenn es um Verhaltensweisen geht, die andere Personen schädigen, wird man diese Geschädigten nicht mit dem Verweis darauf beruhigen können, dass es sich bei dem ihnen entstandenen Schaden um eine insgesamt gesehen zu vernachlässigende Größe handelt ${ }^{41}$.

Auch dann, wenn man das Aufsichts- und das Haftungsrecht nicht als von vornherein ungeeignet einstuft ${ }^{42}$, kommt man nach alledem nicht um die Erkenntnis herum, dass die Androhung und Verhängung strafrechtlicher Sanktionen einerseits und der Einsatz der präventiven Instrumente des Aufsichts- und des Zivilrechts andererseits entgegen landläufiger Vorstellungen nicht in einem Verhältnis des strikten „Entweder-oder“ stehen, sondern es sich tatsächlich um ein Verhältnis des differenzierten „Sowohl-als-auch“ handelt ${ }^{43}$. Dies bedeutet, dass einerseits die Legitimation des Einsatzes strafrechtlichen Zwangs nicht durch den schlichten Verweis auf - theoretisch denkbare und/oder praktisch zur Verfügung stehende - Instrumente anderer Teilbereiche der Rechtsordnung in Frage gestellt werden kann, dass aber andererseits der Einsatz strafrechtlichen

38 Woblers, in: Ackermann/Woblers (Hrsg.), Finanzmarkt ausser Kontrolle?, Zürich 2009, S. 288 f.; vgl. aber auch die sehr viel positivere Einschätzung bei Lüderssen, in: Kempf/Lüderssen/Volk (Anm.17), S. $273 \mathrm{ff} ., 311 \mathrm{ff}$.

39 Tiedemann, Wirtschaftsstrafrecht, 3. Aufl. 2010, Rdn. 63 a; ders., ZStW 87 (1975), S. 253, 266 f.; ablehnend hierzu Böse, EuCLR 2011, 35, 39 f.; Herzog, Gesellschaftliche Unsicherheit und strafrechtliche Daseinsvorsorge, 1991, S.119ff.; Prittwitz, in: Kempf/Lüderssen/Volk (Anm. 17), S. 58 f.; Volk, JZ 1982, 85, 88; Woblers, Deliktstypen (Anm. 12), S. 76 f.; ders., in: von Hirsch/Seelmann/Woblers (Hrsg.), Mediating Principles, 2006, S. $64 \mathrm{ff}$.

40 Böse EuCLR 2011, 35, 39; Haffke, KritV 1991, 165, 173 f.; vgl. auch Kasiske, in: Schünemann (Anm. 1), S. 38/39.

41 Woblers, Mediating Principles (Anm. 39), S. 68.

42 So aber im Hinblick auf den Untreuestraftatbestand Schünemann, StraFo 2010, 1, 2, mit dem Verweis darauf, dass „alle sonst üblichen faktischen und zivilrechtlichen Schutzvorkehrungen gegenüber denjenigen Personen versagen, denen der jederzeitige Zugriff auf das Vermögen eines anderen von Rechts wegen eröffnet ist"; vgl. auch ders., in: Schünemann (Anm.1), S. $85 \mathrm{ff}$. sowie Kasiske, in: Schünemann (Anm.1), S. 39 f. mit dem Hinweis darauf, dass insbesondere zivilrechtliche Schadensersatzansprüche nicht praktikabel seien.

43 Vgl. hierzu bereits Woblers, Deliktstypen (Anm.12), S.77; ders., Mediating Principles (Anm. 39), S. 66. 
Zwangs nur dann legitim ist, wenn dies mit Blick auf die betroffenen Grundrechte als ein legitimer Eingriff begründet werden kann ${ }^{44}$.

Und für den Rechtsanwender gilt: Es ist nicht ersichtlich, mit welcher Begründung man ein Verhalten, das alle Voraussetzungen für die Anwendung einer geltenden Strafnorm erfüllt, als strafrechtlich irrelevant einstufen können soll, „nur“ weil man auch mit anderen Mitteln vorgehen könnte. Wenn dies ein tragfähiges Argument wäre, dann könnte und müsste man zunächst einmal mit Blick auf das zivile Haftungsrecht praktisch dem gesamten Eigentums- und Vermögensstrafrecht einen legitimen Anwendungsbereich absprechen. Man müsste aber wohl darüber hinaus auch die Strafbarkeit wegen Körperverletzung in Frage stellen, da auch hier die zivilrechtliche Reparation von Schädigungen und auch Genugtuungsleistungen denkbar und rechtlich vorgesehen sind ${ }^{45}$.

\section{Die Erfassung des Verhaltens der auf dem Finanzmarkt agierenden Akteure durch die Straftatbestände des Untreuestrafrechts}

Die entscheidende Frage, der wir uns nun endgültig zuwenden können, ist die, ob das konkret in Frage stehende Verhalten der auf dem Finanzmarkt im Vorfeld und im Rahmen der Finanzkrise agierenden Akteure die Voraussetzungen erfüllt, die das Gesetz an eine Bestrafung wegen Untreue $(\mathbb{2} 266 \mathrm{dStGB}$ bzw. $\mathbb{1} 153$ öStGB) oder - in der Diktion des schweizerischen Rechts - wegen ungetreuer Geschäftsbesorgung (Art. 158 schwStGB) knüpft.

Der Untreuestraftatbestand des deutschen Rechts setzt, wie auch die entsprechenden Straftatbestände des österreichischen und des schweizerischen Rechts, grob gesagt ein pflichtwidriges Verhalten voraus. Dieses pflichtwidrige Verhalten muss dann zu einer Schädigung bzw. - in der Diktion des österreichischen und des deutschen Rechts - zu einem Nachteil bei dem zu betreuenden Vermögen führen. Bei alledem muss der Täter ${ }^{46}$ vorsätzlich handeln. Obwohl sich bei allen drei Strafbarkeitsvoraussetzungen Anwendungsprobleme stellen, dürfte bezogen auf die hier in Frage stehenden Fallgestaltungen das entscheidende Problem beim Merkmal der Pflichtwidrigkeit liegen.

Vgl. hierzu bereits Woblers, Mediating Principles (Anm. 39), S. 67 f.; vgl. auch Böse, EuCLR 2011, 35, 40.

45 Der denkbare (Gegen-)Einwand, dass es insoweit auch darum gehen müsse, auch auf zahlungsunfähige Täter einwirken zu können, überzeugt gerade mit Blick auf die vorliegend im Fokus stehenden Fallgestaltungen nicht: Trotz aller Boni dürfte es undenkbar sein, dass irgendein Manager der von der Finanzkrise betroffenen Banken in der Lage ist, den - hier argumendi causa unterstellten -Schaden zu beseitigen, den er seinem Unternehmen durch sein Verhalten zugefügt hat.

46 Dass es sich bei den mit der Anlage und Verwaltung von Fremdvermögen betrauten Mitarbeitern von Banken und anderen mit Finanzmarktdienstleistungen beschäftigten Unternehmen um taugliche Täter handelt, dürfte unbestritten sein und soll hier nicht weiter thematisiert werden. 


\section{Das Merkmal der Pflichtwidrigkeit}

\section{a) Die asymmetrische Akzessorietät des Pflichtwidrigkeitsmerkmals}

Ob ein Verhalten als pflichtgemäß oder aber als pflichtwidrig einzustufen ist, ergibt sich nicht aus dem Strafrecht selbst, sondern aus der Primärrechtsordnung ${ }^{47}$, im vorliegenden Zusammenhang also aus den Bestimmungen des für die Banken geltenden Aufsichtsrechts sowie aus dem Gesellschafts- und sonstigen Zivilrecht. Die Bestimmung der strafrechtlichen Verbotsmaterie kann und darf nicht von der primärrechtlichen Beurteilung des Verhaltens abgekoppelt werden, wobei allerdings festzuhalten bleibt, dass auch die Normen der Primärrechtsordnung nur dann und nur insoweit maßgebend sind, als sie entweder von den am jeweiligen Rechtsverhältnis beteiligten Personen ausdrücklich oder konkludent vereinbart worden sind oder es sich um Normen handelt, deren Verbindlichkeit durch den Gesetzgeber zwingend vorgegeben ist ${ }^{48}$.

Zu klären bleibt, wie sich die Akzessorietät des Untreuestrafrechts zur Primärrechtsordnung auf den Anwendungsbereich der in Frage stehenden Straftatbestände auswirkt. Der soweit ersichtlich weitgehend konsentierte Grundansatz geht dahin, dass das Strafrecht zurückhaltend vorgehen muss, es muss also - wie Rönnau es formuliert hat - die Pflichtwidrigkeit bei der Untreue „restriktiv“ interpretiert werden ${ }^{49}$. Tiedemann propagiert in der Festschrift für Weber „eine in der Tendenz engere Auslegung des Strafrechts im Verhältnis zum Zivilrecht ${ }^{* 50}$, die dann letztlich dazu führen soll, dass die Primärrechtsordnung und das Strafrecht durch zwei konzentrische Kreise zu veranschaulichen sind, wobei das Strafrecht den inneren Ring bildet ${ }^{51}$. Und der BGH hat - jedenfalls zwi-

47 BVerfGE 126, 170, 204 mit zust. Bespr. Wessing/Krawczyk, NZG 2010, 1121, 1122; BGHSt. 47, 187, 197; 47, 295, 297; 55, 288, 300; Dierlamm, in: Münchener Kommentar zum Strafgesetzbuch, \266 Rdn. 152; Saliger, in: Satzger/Schmitt/Widmaier, Kommentrar zum Strafgesetzbuch, \$2 266 Rdn. 31; Hoyer, in: SK StGB, \$266 Rdn. 47; Becker/Walla/Endert, WM 2010, 875, 876; Beulke, Festschrift für Eisenberg, 2009, S. 250; Bosch/Lange, JZ 2009, 225, 226; Brammsen, wistra 2009, 85, 86; Brüning/Samson, ZIP 2009, 1089, 1090; Lüderssen, Festschrift für Eser, 2005, S. 163 f.; Ransiek/Hüls, ZGR 2009, 157, 161 ff.; Rönnau, ZStW 119 (2007), S. 887, 906f.; Tiedemann, Festschrift für Weber, 2004, S. 322 sowie - zum schweizerischen Recht - Donatsch, schwZStrR 120 (2002), S. 1, 7; vgl. auch Schünemann, in: LK, \$266 Rdn. 94 sowie - allgemein zum Wirtschaftsstrafrecht - Haffke, KritV 1991, 165, 174; Prittwitz, in: Kempf/Lüderssen/Volk (Anm. 17), S. 56; a. A. Schünemann, Organuntreue-Der Fall Mannesmann als Exempel, 2004, S. $21 \mathrm{ff}$.

48 Vgl. auch Dierlamm (Anm.47), \266 Rdn.151; Kindhäuser, in: Nomos Kommentar zum Strafgesetzbuch, \266 Rdn.63, 74, 75 a; Schünemann, in: LK (Anm. 47), \266 Rdn. 125 (S. 110); Saliger (Anm. 47), \$266 Rdn. 31 sowie - für das schweizerische Recht - Donatsch, schwZStrR 114 (1996), S.200, 211 f.; ders., schwZStrR 120 (2002), S. 1, 8; Niggli, in: Niggli Wiprächtiger, Basler Kommentar Strafrecht II, 2. Aufl., Bern 2007, Art. 158 Rdn. 57 f.; Stratenwerth/Woblers (Anm. 30), Art. 158 Rdn. 4; Trechsel/Crameri, in: Trechsel et al., Schweizerisches Strafgesetzbuch, Praxiskommentar, Art. 158 Rdn. 9.

49 Rönnau, ZStW 119 (2007), S. 887, 909; so auch Bittmann NStZ 2011, 369, 372; Radtke/Hoffmann, GA 2008, 535, 536.

50 Tiedemann, Festschrift für Weber, 2004, S. 322.

51 Tiedemann, Festschrift für Weber, 2004, S. 323. 
schenzeitlich einmal - die These verfolgt, dass die Pflichtwidrigkeit eine „gravierende" Regelverletzung zur Voraussetzung habe ${ }^{52}$. Die Frage ist nun aber: Was bedeutet das konkret? Sind nur besonders gewichtige Verstöße relevant? Wenn ja: wann ist das der Fall? Oder bedeutet es eine zweite Prüfungsstufe nach der Feststellung der Gesellschaftsrechtswidrigkeit ${ }^{53}$ ? - Wenn ja: was ist genau Funktion und Inhalt dieser Prüfungsstufe?

Richtigerweise ist von einem in der Literatur überwiegend als Prinzip der asymmetrischen Akzessorietät bezeichneten Modell ${ }^{54}$ auszugehen. Unter Zugrundelegung dieses Modells gilt zunächst einmal, dass, wenn die Primärrechtsordnung bestimmte Verhaltensweisen für zulässig erklärt, das Strafrecht diese Wertung nicht konterkarieren darf: Was zivilrechtlich erlaubt ist, kann strafrechtlich nicht verboten sein ${ }^{55}$. Dieser Grundsatz gilt im Übrigen nicht nur für die Untreue, sondern z. B. auch für die Buchführungsdelikte. Schröder hat zutreffend darauf hingewiesen, dass die Eröffnung großer Bewertungsspielräume im Bilanzrecht dem Strafrecht „das Schwert aus der Hand [schlägt], weil das Strafrecht nicht bestrafen kann, was das Bilanzrecht zulässt" ${ }^{\text {"56. }}$

Zum zweiten gilt aber auch: Wenn die Primärrechtsordnung bestimmte Verhaltensweisen für unzulässig erklärt, dann hat das Strafrecht auch diese Wertung zu übernehmen. In diesen Fällen kann - wie der BGH in der Mannesmann-Entscheidung klargestellt hat ${ }^{57}$ - nicht noch als ein zusätzliches Kriterium hinzu kommen, dass es sich um eine gravierende Pflichtverletzung handeln muss ${ }^{58}$. Die Frage ist allenfalls, ob aufgrund strafrechtsspezifischer Gesichtspunkte trotz der

52 BGHSt. 47, 148, 150; 47, 187, 197; BGH NStZ 2011, 403, 405; vgl. hierzu Dierlamm (Anm. 47), \$266 Rdn. 154 ff.; Kindhäuser (Anm. 48), \$266 Rdn. 75 a f.; Saliger (Anm. 47), \$266 Rdn. 40 ff.; Hoyer (Anm. 47), \$266 Rdn. 54 ff.; Saliger, HRRS 2006, 10, 19 f. sowie Theile, ZIS 2011, $616 \mathrm{f}$. sowie $623 \mathrm{ff}$.

53 So Dierlamm, StraFo 2005, 397, 402 ff.; kritisch hierzu Schünemann, Organuntreue (Anm. 47), S. $27 \mathrm{ff}$.

54 Vgl. Dierlamm, StraFo 2005, 397, 398; Lüderssen, Festschrift für Eser, 2005, S. 163, 170; ders., Festschrift für Lampe, 2003, S. 727, 729; ders., in: Kempf/Lüderssen/Volk (Anm. 17), S. 299; Saliger(Anm. 47), \266 Rdn. 31; Dierlamm (Anm. 47), \$266 Rdn. 153; Hoyer (Anm. 47), \$266 Rdn. 47; vgl. auch BVerfGE 126, 170, 198; Beulke, Festschrift für Eisenberg, 2009, S. 252 f.

55 Dierlamm (Anm. 47), \$266 Rdn. 152; Saliger (Anm. 47), \$266 Rdn. 31; Becker/Walla/Endert, WM 2010, 875, 878; Beulke, Festschrift für Eisenberg, 2009, S. 251; Bosch/Lange, JZ 2009, 225, 226; Dierlamm, StraFo 2005, 397 f.; Günther, Festschrift für Weber, 2004, S. 314; Lüderssen, Festschrift für Eser, 2005, S.163, 170; ders., in: Kempf/Lüderssen/Volk (Anm. 17), S. 299; Radtke/Hoffmann, GA 2008, 535, 538; Rönnau, NStZ 2006, 218, 220; Tiedemann, Festschrift für Weber, 2004, S. 323; Volk, Festschrift für Hamm, 2008, S. 803, 804; Zech, Untreue durch Aufsichtsratsmitglieder einer Aktiengesellschaft, 2007, S.212; vgl. auch Brammsen, wistra 2009, 85, 87; zum schweizerischen Recht: Donatsch, Strafrecht III, Delikte gegen den Einzelnen, 9. Aufl., Zürich 2008, S. 278; ders., schwZStrR 120 (2002), S. 1, 17/18.

56 Schröder, Kriminalistik 2009, 12, 13; vgl. zu den insoweit auftretenden Schwierigkeiten auch bereits Ransiek, WM 2010, 869, 870 ff.; Rönnau, in: Schünemann (Anm. 1), S. 51 ff.

57 BGHSt. 50, 331, 343 ff. mit zust. Bespr. Ransiek, NJW 2006, 814; vgl. auch Kubiciel, NStZ 2005, 353, $358 \mathrm{f}$.

58 Kritisch zur Aufgabe dieses Kriteriums als zusätzliches Merkmal zur Bestimmung einer (auch) strafrechtlich relevanten Pflichtwidrigkeit Beulke, Festschrift für Eisenberg, 2009, S. $253 \mathrm{ff}$. 
zu konstatierenden Pflichtwidrigkeit des Verhaltens im Ergebnis doch kein Strafvorwurf zu erheben ist. So kann z. B. eine teleologische Reduktion des strafrechtlich relevanten Bereichs dann geboten sein, wenn sich die Anwendung strafrechtlichen Zwangs im Hinblick auf das geschützte Rechtsgut als unverhältnismäßig oder aus sonstigen Gründen verfehlt erweist. Bezogen auf den Untreuestraftatbestand muss es sich z. B. bei der die Pflichtwidrigkeit begründenden Normverletzung im Hinblick auf die Ratio legis der Norm um eine vermögensschützende Norm handeln 59 .

Das eigentliche Problem besteht nun darin, dass auch die Primärrechtsordnung eher selten wirklich klare Wertungen zur Verfügung stellt. Tatsächlich sind die Vorgaben der Primärrechtsordnung in der Regel eher vage und/oder umstritten $^{60}$. In diesem Zusammenhang müssen nun zwei zentrale Probleme gelöst werden: Zunächst einmal muss geklärt werden, wie mit Regelungen umgegangen wird, deren Auslegung umstritten ist - was ja auch außerhalb des Strafrechts nicht der Ausnahme-, sondern eher der Regelfall ist. Darüber hinaus stellt sich - zweitens - die Frage, wer anhand welcher Maßstäbe die strafrechtlich relevanten Grenzen bestimmt.

Im Ergebnis wird man nicht daran vorbei kommen, dass die Kompetenz zur Bestimmung dessen, was zu gelten hat, nur bei den Gerichten liegen kann - und zwar, soweit es um die Bestimmung des strafrechtlich relevanten Verhaltens, bei den Strafgerichten ${ }^{61}$. Zur Begründung dieser These ist zunächst darauf zu verweisen, dass man die Zivilrechtsakzessorietät einer strafrechtlichen Norm nicht verwechseln darf mit Zivilgerichtsakzessorietät oder gar Zivilrechtslebrerakzessorietät ${ }^{62}$.

Würde man - wie es in der Literatur zum Teil vertreten wird - darauf abstellen wollen, dass die Pflichtwidrigkeit eines Verhaltens schon dann zu verneinen ist, wenn auch nur ein Autor das in Frage stehende Verhalten als pflicht-

59 BVerfGE 126, 170, 220; BGHSt. 47, 295, 297; Brammsen, wistra 2009, 85, 87; Brüning/Samson ZIP 2009, 1089, 1091; Günther, Festschrift für Weber, 2004, S. 316 f.; Kubiciel, NStZ 2005, 353, 355 und 360; Schünemann, NStZ 2006, 196, 198; vgl. auch Saliger (Anm. 47), \$266 Rdn. 32 und 35; Hoyer (Anm. 47), \$266 Rdn. 51 sowie als aktuelle Anwendungsfälle aus der Rechtsprechung BGHSt. 55, 288, 300 ff. zu den Normen betreffend die Wahl der Betriebsräte; BGHSt. 55, 266, $277 \mathrm{f}$. zum vermögensschützenden Charakter der handelsrechtlichen Buchführungspflicht; zum schweizerischen Recht vgl. Donatsch (Anm. 55), S. 277.

60 Vgl. Hermes, in: Kempf/Lüderssen/Volk (Anm. 1), S. 44 ff.: auch das Wirtschaftsverwaltungsrecht könne keine scharfen Abgrenzungen gewährleisten; zur Entwicklung des Wirtschaftsverwaltungsrechts hin zu einem sich ständig ausdifferenzierenden und auf neue Formen zurückgreifenden Regulierungsrecht vgl. Uwer, in: Kempf/Lüderssen/Volk (Anm. 17), S. 127 ff.

61 So auch bereits Beulke, Festschrift für Eisenberg, 2009, S. 251; Rönnau, NStZ 2006, 218, 220; ders., ZStW 119 (2007), S. 887, $913 \mathrm{ff.}$

62 Hoyer (Anm. 47), \$266 Rdn. 48; Ransiek/Hüls, ZGR 2009, 157, 172; Rönnau, NStZ 2006, 218, 220; ders., ZStW 119 (2007), S. 887, 913; vgl. auch Lüderssen, Festschrift für Schroeder, 2006, S. 569, 574: „Akzessorietät ist aber prima vista nur gesetzesbezogen; sie gilt nicht ohne weiteres auch für die Auslegung der Gesetze. In der Tat kann ja weder die Autorität irgendeines wissenschaftlichen Autors oder eines obersten Gerichts, noch die herrschende Meinung massgebend sein.“ 
gemäß einstufen würde ${ }^{63}$, sollte man sich den ganzen mit der Strafverfolgung verbundenen Aufwand lieber gleich sparen, da sich auch bezogen auf die Primärrechtsordnung praktisch wohl immer wenigstens eine Stimme finden lassen wird, nach der sich das in Frage stehende Verhalten als mehr oder weniger gut vertretbar einstufen lässt. Und wenn man auf die „herrschende Lehre“ oder die „herrschende Meinung“ abstellen will, läuft man in die allenfalls noch für soziologische oder psychologische Feldstudien interessante Problematik hinein, wie man überhaupt bestimmen will, was das ist: eine „herrschende“ Meinung.

Gewichtiger ist der Einwand, dass aus der Existenz von Meinungsstreitigkeiten bei der Auslegung des relevanten Primärrechts der Schluss zu ziehen sei, dass die an diese Normen anknüpfende Strafnorm den Anforderungen des strafrechtlichen Bestimmtheitsgebotes nicht genügen könne ${ }^{64}$. Dass das BVerfG in seiner Entscheidung zur strafrechtlichen Bestimmtheit des deutschen Untreuestraftatbestandes die Konkretisierung einer für sich gesehen im Hinblick auf Art. 103 Abs. 2 GG zu unbestimmten Norm durch eine fallgruppenorientierte Rechtsprechung der Fachgerichte akzeptiert hat ${ }^{65}$, kann man mit guten Gründen als verfassungsrechtlich zweifelhaft ansehen ${ }^{66}$. Auf einem anderen Blatt steht, dass die Rechtsprechung bei der Anwendung von Normen gar nicht anders verfahren kann und dieses Vorgehen damit stets notwendig ist - auch dann, wenn man es mit einer verfassungsrechtlich hinreichend bestimmten Norm zu tun hat. Und schließlich wird man nicht ohne weiteres annehmen können, dass sich die Unbestimmtheit des Untreuestraftatbestands tatsächlich signifikant von anderen Normen unterscheidet, die als höchst unbestimmt angesehen werden müssen, ohne dass sich hier die aus der Akzessorietät ergebenden Auslegungsprobleme stellen $^{67}$. Legt man die Maßstäbe an, die das BVerfG für das Bestimmtheitsgebot des Art. 103 Abs. 2 GG entwickelt hat, wird man bezweifeln müssen, dass die Akzessorietät der Norm deren Bestimmtheit in Frage stellt. Wenn es derartige besondere Bestimmungen im nationalen Recht nicht gibt oder - wie z. B. im

So dezidiert Zech (Anm. 55), S. 216, die dann allerdings eine Ausnahme für die Fallgestaltungen anerkennen will, bei denen eine höchstrichterliche Entscheidung vorliegt.

64 Lüderssen, Festschrift für Eser, 2005, S.163, 178 f.; wohl weniger strikt ders., Festschrift für Schroeder, 2006, S. 569, 577: Die Auslegung der für die Anwendung des Strafrechts relevanten Normen der Primärrechtsordnung müsse „eine gewisse Evidenz und Eindeutigkeit aufweisen, weil nur so der aus Art. 103 Abs. 2 GG folgende Grundsatz der Voraussehbarkeit strafrechtlicher Verfolgung valutiert werden kann“. Vgl. auch Dierlamm (Anm. 47), $\$ 266$ Rdn. 152; ders., StraFo 2005, 397, 400.

65 BVerfGE 126, 170, $197 \mathrm{f}$. und $209 \mathrm{ff}$.

66 Kritisch hierzu Wessing/Krawczyk, NZG 2020, 1121, 1122 f.; Krüger, NStZ 2011, 369, 374; Safferling, NStZ 2011, 376, 377; Steinberg/Dinter, JR 2011, 224, 225.

67 Als Beispiele, die allen drei deutschsprachigen Rechtsordnungen gemein sind, kann auf die Straftatbestände der Beleidigung ( $\$ 185$ StGB, $\$ 115$ öStGB und Art. 177 schwStGB) und der Nötigung ( $\$ 240$ StGB, $\$ 105$ öStGB und Art. 181 schwStGB) verwiesen werden. Als weiteres eindrückliches Beispiel aus dem schweizerischen Recht ist der Tatbestand des Mordes (Art. 112 schwStGB) zu nennen: „Handelt der Täter besonders skrupellos, sind namentlich sein Beweggrund, der Zweck der Tat oder die Art der Ausführung besonders verwerflich, so ist die Strafe lebenslängliche Freiheitsstrafe oder Freiheitsstrafe nicht unter zehn Jahren.“ 
schweizerischen Recht - die Strafgerichte sogar ausdrücklich angehalten sind, eine Strafnorm selbst dann anzuwenden, wenn sich diese aus ihrer Sicht als verfassungswidrig darstellt ${ }^{68}$, hat sich die Frage nach etwaigen Konsequenzen der Unterbestimmtheit des Untreuestraftatbestands bereits im Ansatz erledigt.

Aber selbst dann, wenn eine bestimmte Rechtsfrage durch eine ständige Rechtsprechung der Zivilgerichte - jedenfalls für die Praxis - geklärt ist, ändert dies nichts daran, dass die Rechtsprechung über strafrechtliche Normen der Strafjustiz anvertraut ist. Selbstverständlich kann sich die Strafjustiz der durch die Zivilgerichte vertretenen Lösung anschließen und sie wird dies in der Regel auch tun - und dies aus guten Gründen. Zwingend ist dies aber nicht ${ }^{69}$. Die Auslegung strafrechtlicher Normen liegt in der Kompetenz des Strafrichters und nicht des Zivilrichters, es gilt, was aus der Sicht des Strafrichters die „richtige " Lösung ist ${ }^{70}$. Der Strafrichter ist allerdings gehalten, sich mit den in der Lehre und in der Zivilrechtsprechung vertretenen Auffassungen auseinanderzusetzen, und er hat darzutun, warum er der von ihm zugrunde gelegten Auslegung folgt ${ }^{71}$.

Der Strafrichter ist bei der Auslegung des objektiven Tatbestands nicht gehalten, die für den Beschuldigten bestmögliche Lösung zu präferieren ${ }^{72}$. Der Strafrichter muss aber bei der Interpretation der die Verbotsmaterie mitkonstituierenden Normen der Primärrechtsordnung den allgemein für das Strafrecht geltenden Auslegungsmaximen genügen, wie z.B. dem strafrechtsspezifischen Bestimmtheitsgebot und dem Analogieverbot ${ }^{73}$. Wenn z. B. die Primärrechtsordnung mit Vermutungen arbeitet oder ein Umgehungsverbot statuiert, dann

68 Aus der Regelung des Art. 190 schwBV („Bundesgesetze und Völkerrecht sind für das Bundesgericht und die anderen rechtsanwendenden Behörden massgebend.") folgt, dass die Strafgerichte der Schweiz Straftatbestände des Bundesrechts auch dann anzuwenden haben, wenn sie diese für verfassungswidrig halten. Eine Ausnahme gilt nach der Rechtsprechung des schweizerischen Bundesgerichts allein für die Normen des Bundesrechts, die (auch) gegen die EMRK und damit gegen völkerrechtliche Vorgaben verstoßen (sog. Schubert-Praxis); vgl. Hangartner, in: Ehrenzeller et al. (Hrsg.), Die schweizerische Bundesverfassung, 2. Aufl., Zürich 2008, Art. 190 Rdn. 32.

69 Anderer Ansicht Zech (Anm. 55).

70 A. A. Haffke, KritV 1991, 165, 173 f.: Es sei nicht Sache des Strafrechts, zu zivilrechtlichen Fragen Stellung zu nehmen und diese durch Kriminalisierung wirkungsträchtig zu entscheiden.

$71 \mathrm{Zu}$ den Anforderungen an die Begründung richterlicher Entscheidungen vgl. - mit besonderem Bezug auf die Revisionsentscheidungen des BGH - Fezer, HRRS 2010, 281, 282 ff. sowie Woblers, JZ 2011, 78, $79 \mathrm{ff}$.

72 So aber im Ergebnis Dierlamm, StraFo 2005, 397, 401; Haffke, KritV 1991, 165, 173 ff.; Lüderssen, Festschrift für Eser, 2005, S. 163, 170; ablehnend hierzu Rönnau ZStW 119 (2007), S. 887, 915 mit dem zutreffenden Hinweis, dass dieser Ansatz letztlich auf eine „in dubio pro reo-Lösung bei der Auslegung von Rechtsfragen“ hinauslaufen würde; vgl. auch Vogel/Hocke, JZ 2006, 568, 569.

73 Hoyer (Anm. 47), \$266 Rdn. 50; Lüderssen StV 2009, 486, 492; ders., Festschrift für Eser, 2005, S. 163, 170 Fn. 50; ders. Festschrift für Schroeder, 2006, S. 569, 575 f.; ders., in: Kempf/Lüderssen/Volk (Anm. 1), S. 230; Otto, Festschrift für Gitter, 1995, S. 715, 716; Tiedemann, Festschrift für Weber, 2004, S. 322; vgl. auch Ransiek/Hüls, ZGR 2009, 157, 174. 
wird das Strafrecht dem nicht ohne weiteres folgen können ${ }^{74}$. Und bezogen auf das Merkmal der Pflichtwidrigkeit bleibt festzuhalten: Auch wenn es gesellschaftsrechtlich gesehen in Ordnung sein mag, die Grenze des zulässigen Verhaltens mit dem Maßstab des „ordentlichen Kaufmanns“ zu bestimmen; strafrechtlich gesehen geht es nicht an, sich auf schwammige Formeln zurückzuziehen wie die, der Täter habe „nach Art eines Spielers bewusst und entgegen den Regeln kaufmännischer Sorgfalt" gehandelt ${ }^{75}$. Strafrechtlich gesehen wird man in derartigen Situationen einen dem Bestimmtheitsgebot geschuldeten Sicherheitspuffer einbauen müssen ${ }^{76}$, womit man dann bei der wohl legitimen Funktion dessen angekommen ist, was man mit dem vom BGH vorgeschlagenen Kriterium der „gravierenden“ Pflichtverletzung erreichen kann" ${ }^{77}$, das man dahingehend zu interpretieren hat, dass ein „eindeutiger“ bzw. „evidenter“ Pflichtenverstoß $\mathrm{zu}$ fordern ist ${ }^{78}$. Aus alledem folgt, dass wir mit dem Phänomen der Normspaltung konfrontiert sein können: Der Bereich des strafrechtlich Pflichtwidrigen kann und wird hinter dem des zivilrechtlich Pflichtwidrigen zurück bleiben $^{79}$.

Besondere Probleme stellen sich schließlich noch beim Umgang mit den Generalklauseln der Primärrechtsordnung, wie z.B. der Business Judgment Rule $^{80}$, nach der ein Vorstandsmitglied für eine unternehmerische Entscheidung dann nicht haftet, wenn es auf der Grundlage angemessener Informationen ge-

74 Vgl. Otto, Festschrift für Gitter, 1995, S. 715, $723 \mathrm{ff}$.

75 So aber BGH, NJW 1974, 1234, 1236; NStZ 1990, 437; StV 2004, 424, 425; ablehnend BVerfGE 126, 170, 227 ff.; Park/Rütters, StV 2011, 434, 439.

76 So im Ergebnis auch Hoyer (Anm. 47), \266 Rdn. 50; Beulke, Festschrift für Eisenberg, 2009, S. 252; Bittmann, NStZ 2011, 361, 365; vgl. auch Spindler, in: Kempf/Lüderssen/Volk (Anm. 1), S. 97 f.; Vogel/Hocke, JZ 2006, 568, 569; kritisch zur Unbestimmtheit der von der Rechtsprechung verwendeten Formel auch Deiters, in: Kempf/Lüderssen/Volk (Anm. 1), S. 137.

77 So auch Becker/Walla/Endert, WM 2010, 875, 878; Brammsen, wistra 2009, 85, 88; Ransiek/ Hüls, ZGR 2009, 157, 170 f.; Schünemann, NStZ 2005, 473, 475 f.; ders., NStZ 2006, 196, 197 ff.; ders., in: Schünemann (Anm. 1), S. 93; Tiedemann, Festschrift für Weber, 2004, S. 322; vgl. auch Rönnau ZStW 119 (2007), S. 887, 910 f.; ders., ZStW 122 (2010), S. 299, 304 f.: Dass es sich um eine gravierende Verletzung gesellschaftsrechtlicher Pflichten handele, ergebe sich aus der Überschreitung des durch die Primärrechtsordnung eröffneten weiten Ermessensspielraums; vgl. im Ergebnis auch Günther, Festschrift für Weber, 2004, S. 314 ff.; Theile, ZIS 2011, 616, 623 ff.; vgl. aber auch Bosch/Lange, JZ 2009, 225, 235 f.: Der BGH habe lediglich den differenzierten Haftungsmaßstab des $\int 93$ AktG für das Strafrecht fruchtbar gemacht. Eine Beschränkung auf evidente Fälle sei hiermit nicht verbunden.

78 BVerfGE 126, 170, 217 f. mit zust. Bespr. Saliger, NJW 2010, 3195, 3197; BGHSt. 55, 288, 300; vgl. auch Brüning/Samson ZIP 2009, 1089, 1093 f.; Rönnau, NStZ 2006, 218, 220; ders., ZStW 119 (2007), S. 887, 917; Saliger HRRS 2006, 10, 20; Tiedemann, Festschrift für Tröndle, 1989, S. 328; vgl. auch Kubiciel, NStZ 2005, 353, 360; kritisch gegenüber diesem Kriterium Krüger, NStZ 2011, 369, 374; Safferling, NStZ 2011, 376, 377.

79 Hierzu auch bereits BGHSt. 24, 54, 61 f.; Hoyer (Anm. 47), \266 Rdn. 50; Lüderssen, Festschrift für Schroeder, 2006, S. 569, 574 ff.; Otto, Festschrift für Gitter, 1995, S. 715, 716 ff.; Tiedemann, Festschrift für Weber, 2004, S. 322 f.

80 Im deutschen Recht ist die Business Judgment Rule in \$93 Abs. 1 Satz 2 dAktG ausdrücklich kodifiziert worden. Im österreichischen und schweizerischen Recht wird die Business Judgment Rule jedenfalls der Sache nach ebenfalls anerkannt; vgl. - für die Schweiz - Böckli, Schweizer Aktienrecht, 4. Aufl., Zürich 2009, Rdn. 581 ff. sowie - für Österreich - Kalss, in: 
handelt hat und es aufgrund dieser Informationen davon ausgehen durfte, zum Wohle der Gesellschaft zu handeln ${ }^{81}$. Wenn die Business Judgment Rule den Mitgliedern der Geschäftsleitung eines Unternehmens einen nicht unerheblichen Spielraum eröffnet, stellt sich die Frage, wann die Grenze des Spielraums überschritten ist und wer bestimmt, dass das so ist.

Im Ergebnis ist klar, dass auch diese Entscheidung wieder bei den Strafgerichten liegen muss, die sich aber der in diesem Zusammenhang drohenden besonderen Gefahren bewusst sein müssen. Da besteht zunächst einmal Gefahr, dass die Gerichte anfangen, Politik zu machen. Wenn man beispielsweise Unternehmensinteressen als auch gemeinwohlorientiert interpretiert ${ }^{82}$, kann man abgesehen davon, dass sich die Frage stellt, wie man die Berücksichtigung der Interessen anderer Stakeholder und/oder der Gesellschaft als solcher mit der Struktur des Untreuestraftatbestands als Vermögensdelikt vereinbaren soll ${ }^{83}$ praktisch beliebige Ergebnisse produzieren ${ }^{84}$. Vor allem aber besteht die Gefahr, dass die Sicht ex ante mit der Sicht ex post vermengt wird, was dann zu einem sog. Rückschaufehler führen kann ${ }^{85}$.

Beispielhaft und bezogen auf die hier interessierende Fallgestaltung der Finanzkrise: Ex post betrachtet dürfte uns allen nun klar sein, dass das System der Verbriefungen nicht funktionieren konnte, jedenfalls nicht auf Dauer ${ }^{86}$. Die Frage ist nun aber, ob dies auch dem einzelnen Akteur in seiner konkreten Handlungs- und Entscheidungssituation klar sein musste. Tatsächlich wird man hier auf das Wissen im jeweiligen Tatzeitpunkt abstellen und damit eine Zeitphase vor und nach einem bestimmen Ereignis unterscheiden müssen, wobei bezogen auf die aktuelle Finanzkrise entweder auf das Publikwerden der Subprime-Krise in den USA (ca. Mitte 2007) oder aber - allerspätestens - auf die Pleite von Lehmann Brothers (September 2008) abzustellen sein dürfte ${ }^{87}$. Dass das System der Verbriefung schon rein abstrakt gesehen auf Dauer nicht funktionieren konnte, wird demgegenüber nicht ausreichen. Angesichts dessen, dass nicht nur das Eingehen, sondern auch das Nichteingehen eines Risikos pflichtwidrig sein kann ${ }^{88}$,

Kalss/Nowotny/Schaner, Österreichisches Gesellschaftsrecht, Wien 2008, Rdn. AG 389 sowie OG 2/297.

81 Bosch/Lange, JZ 2009, 225, 229 ff.; Brüning/Samson, ZIP 2009, 1089, 1092; Säcker, in: Kempf/ Lüderssen/Volk (Anm. 1), S. 120 ff.; Spindler, NZG 2010, 281, 283 f.

82 Vgl. hierzu die Beiträge von Suchanek, Spindler und Forstmoser, in: Kempf/Lüderssen/Volk (Anm. 1), S. $47 \mathrm{ff} ., 71 \mathrm{ff} ., 107 \mathrm{ff}$.

83 Vgl. Bosch/Lange, JZ 2009, 225, $232 \mathrm{f}$.

84 Zur Unklarheit der Konsequenzen einer Gemeinwohlorientierung vgl. Lüderssen, StV 2009, 486, 490 f.; ders., in: Kempf/Lüderssen/Volk (Anm. 17), S. 278 ff.; Salditt, in: Kempf/Lüderssen/ Volk (Anm. 17), S. $113 \mathrm{ff}$.

85 Vgl. Brüning/Samson, ZIP 2009, 1089, 1092; Deiters, in: Kempf/Lüderssen/Volk (Anm. 1), S. 137; Fleischer, NJW 2010, 1504, 1506; Park/Rütters, StV 2011, 434, 438; Schröder, Handbuch (Anm. 1), Rdn. 1168; ders., NJW 2010, 1169, 1171; Spindler, NZG 2010, 281, 284; vgl. auch zum schweizerischen Recht - Donatsch (Anm. 55), S.277; ders., schwZStrR 120 (2002), S. 1, 8.

86 Kasiske, in: Schünemann (Anm. 1), S. 22.

87 Für letzteres z. B. Bittmann, NStZ 2011, 361, 362.

88 Bittmann, NStZ 2011, 361, 364. 
kann es Situationen geben, in denen das Verhalten unter Untreuegesichtspunkten selbst dann nicht pflichtwidrig ist, wenn der Täter an einem Schneeballsystem mitwirkt: wenn und soweit der Täter bei pflichtgemäßer Beurteilung seines Verhaltens mit ausreichender Sicherheit davon ausgehen durfte, dass er mit seinem Verhalten dem von ihm zu betreuenden Vermögen keinen Schaden zufügen, sondern dieses erhalten oder gar mehren werde, ist eine untreuerelevante Pflichtwidrigkeit nicht gegeben ${ }^{89}$. Ist diese Voraussetzung gegeben, kann auch weder aus der - ex post betrachtet - gegebenen Gefährdung noch aus dem tatsächlichen Eintritt eines Schadens auf die Pflichtwidrigkeit rückgeschlossen werden ${ }^{90}$ : dies wäre dann nämlich genau der Rückschaufehler, der vermieden werden muss.

\section{b) Anwendung auf das Verbalten im Vorfeld und/oder im Rabmen der Finanzkrise}

Im Rahmen der untreuestrafrechtlichen Bewertung der aktuellen Finanzkrise stellt sich bezogen auf das Straftatmerkmal der Pflichtwidrigkeit die Frage, ob das Mitmachen beim System der Verbriefungen Grenzen überschritten hat, die sich aus dem zugrunde liegenden Rechtsverhältnis ergeben ${ }^{91}$. Eine Pflichtwidrigkeit ist dann zu bejahen, wenn der Täter mit seinem Verhalten von wirksam vereinbarten Vorgaben abgewichen ist oder die für sein Vorgehen nach den Vorgaben des Rechtsverhältnisses zum Vermögensinhaber notwendigen Voraussetzungen nicht gegeben waren $^{92}$.

Lassen sich dem konkreten Rechtsverhältnis keine ausdrücklichen Regelungen entnehmen, stellt sich die Frage, ob die Pflichtwidrigkeit im Anschluss an die einschlägige Rechtsprechung des BGH aus der Gefahr der Existenzvernichtung abgeleitet werden kann. Gegen diesen Ansatz wird in der Literatur der Einwand erhoben, dass Gesellschaften auch nicht davor geschützt sind, dass die Gesellschafter sie liquidieren ${ }^{93}$, womit dann die entscheidende Frage die ist, ob das Verbot der Existenzvernichtung eine Grenze der Dispositionsbefugnis über das Gesellschaftsvermögen begründet. Der BGH geht davon aus, dass die Gesellschafter über das Gesellschaftsvermögen nicht verfügen dürfen, wenn dadurch eine konkrete Existenzgefährdung für die Gesellschaft entsteht, was jedenfalls bei einem Angriff auf das geschützte Stammkapital der Fall sei ${ }^{94}$. Die entschei-

So wohl auch Schünemann, in: Schünemann (Anm. 1), S. 94 f., für nur kurzfristige, das eigene Überleben nicht gefährdende Engagements; Brüning/Samson, ZIP 2009, 1089, 1094, verneinen für derartige Fallgestaltungen das Vorliegen eines (Gefährdungs-)Schadens. BVerfGE 126, 170, 219; BGHSt. 46, 30, 34.

91 Bittmann, NStZ 2011, 361, 364; Park/Rütters, StV 2011, 434, 437.

92 Bittmann, NStZ 2011, 361, 362; vgl. diesbezüglich zu den Fällen der Sächsischen Landesbank und der IKB: Florstedt, AG 2010, 315, $316 \mathrm{f}$.

93 Volk, Festschrift für Hamm, 2008, S. 803, 812

94 Vgl. BGHSt. 34, 379, 385 ff.; 35, 333, 336 ff.; 49, 147, 157 ff.; 54, 52, 58 ff. sowie Achenbach, Festgabe BGH, Band IV, 2000, S. 596 ff.; Beulke, Festschrift für Eisenberg, 2009, S. 256 ff.; Radtke/Hoffmann, GA 2008, 535, 538; Ransiek/Hüls, ZGR 2009, 157, 178 f.; Schünemann, Organuntreue (Anm. 47), S. 30 ff. ; vgl. auch - bezogen auf die Rechtslage in der Schweiz BGE 117 IV 259, 266 ff.; Stratenwerth/Jenny/Bommer (Anm. 30), \$19 Rdn. 25; vgl. aber auch 
dende Frage ist, ob man dem folgen will und - wenn ja - ob es darüber hinaus noch weitere Fälle gibt, in denen man von einer relevanten Existenzgefährdung ausgehen muss oder ausgehen darf. Entscheidend ist, ob man eine - konkludent zum Gegenstand des Rechtsverhältnisses gewordene - Pflicht des Vorstands und der sonstigen Mitglieder der Geschäftsleitung anerkennt, wirtschaftlich evident unvertretbare Risiken zu unterlassen bzw. für den Bestand und die dauerhafte Rentabilität des Unternehmens zu sorgen ${ }^{95}$.

Tatsächlich wird man eine den Mitgliedern der Geschäftsleitung obliegende Pflicht zur Vermeidung existenzgefährdender Risiken anerkennen müssen. Angesichts dessen, dass die Mitglieder der Geschäftsleitung einer Bank gar nicht umhin können, gewisse Risiken einzugehen, kann es diesbezüglich aber von vornherein nur darum gehen, übermäßige Risiken zu vermeiden ${ }^{96}$. Die Unvertretbarkeit des eingegangenen Risikos kann sich daraus ergeben, dass der Täter es unterlässt, die zur Beherrschung des Risikos notwendigen Rücklagen zu bilden ${ }^{97}$, und/oder er durch die Auslagerung der Geschäfte/Risiken in andere Gesellschaften die Kontrolle über das weitere Geschehen verliert ${ }^{98}$. Die Unvertretbarkeit des Risikos kann sich aber auch aus der Undurchsichtigkeit eines Geschäfts und der daraus resultierenden Unkalkulierbarkeit des Risikos ergeben ${ }^{99}$. Die Annahme einer Pflichtwidrigkeit liegt hier insbesondere dann nahe, wenn es sich bei dem eingegangenen Risiko um ein potenziell existenzgefährdendes (Klumpen-)Risiko handelt ${ }^{100}$. Insoweit bleibt dann tatsächlich ein Rest-

Kasiske, JR 2011, 235, 236 ff., mit dem Hinweis darauf, dass unklar ist, ob der BGH auf die Interessen der Gläubiger oder aber auf eigenständige Interessen der Gesellschaft abstelle. Ablehnend zur Vermögensbetreuungspflicht des GmbH-Gesellschafters: Livonius, wistra 2009, 91, $93 \mathrm{ff}$.

95 So BGHSt. 49, 147, 158; Brüning/Samson, ZIP 2009, 1089, 1092 f.; Kasiske, in: Schünemann (Anm. 1), S. 23 ff.; Lutter, ZIP 2009, 197, 199; Radtke/Hoffmann, GA 2008, 535, 541 sowie 547 ff.; Schröder, Handbuch (Anm. 1), Rdn. 1161 f.; vgl. auch Schünemann, in: LK (Anm. 47), $\$ 266$ Rdn. 94: wo kein eindeutiges Verbot feststellbar sei, bleibe in der Regel das Verbot, den Geschäftsherrn zu schädigen; zustimmend Perron, in: Schönke/Schröder, \$266 Rdn. 36; ablehnend gegenüber einem allgemeinen Schädigungsverbot Saliger (Anm. 47), $\$ 266$ Rdn. 43; Dierlamm (Anm. 47), \$266 Rdn. 166; vgl. auch Donatsch, schwZStrR 114 (1996), S. 200, 212; für einen Überblick über den diesbezüglichen Meinungsstand im deutschen Gesellschaftsrecht vgl. Florstedt, AG 2010, 315, 319; Redeke, ZIP 2010, $159 \mathrm{f}$.

96 Vgl. hierzu - aus zivilrechtlicher Sicht-Fleischer NJW 2010, 1504, 1505 f.; Florstedt, AG 2010, 315, 320 ff.; Hellwig (Anm. 1), E 35; Redeke, ZIP 2010, 159, 160 ff.; Spindler, NZG 2010, 281, 284.

97 BGH NStZ 2011, 403, 406 mit einer im konkreten Fall überflüssigen und in der Sache hoch problematischen Aufgabe des Erfordernisses der Unmittelbarkeit des Schadens.

98 Vgl. Spindler, NZG 2010, 281, 285.

99 Park/Rütters, StV 2011, 434, 439; vgl. auch - zur Bedeutung des Verzichts auf eine vernünftige Risikoeinschätzung und Risikokontrolle - Fleischer NJW 2010, 1504, 1506.

100 Schröder, Handbuch (Anm.1), Rdn.1162, 1164 ff.; ders., NJW 2010, 1169, 1172; vgl. auch Kasiske, in: Schünemann (Anm. 1), S. 23 ff.; Bittmann, NStZ 2011, 369, 365; Park/Rütters, StV 2011, 434, 438; Schünemann, in: Schünemann (Anm. 1), S. 91 f.; vgl. auch - aus gesellschaftsrechtlicher Sicht - Fleischer NJW 2010, 1504 f.; Lutter, ZIP 2009, 197, 199; Spindler, NZG 2010, 281, 284 sowie OLG Düsseldorf, AG 2010, 126, 129 mit krit. Bespr. Florstedt, AG $2010,315,320$. 
bestand von Fallgestaltungen, bei denen zwar nicht der Schaden die Pflichtwidrigkeit begründet ${ }^{101}$, wohl aber das dem in Frage stehenden Verhalten inhärente Schädigungspotenzial ${ }^{102}$.

Das Vorliegen einer Pflichtwidrigkeit kann für die vorliegend relevanten Fallgestaltungen weder mit dem schlichten Verweis auf die Ratings der in Frage stehenden Papiere in Abrede gestellt werden ${ }^{103}$, noch mit dem Hinweis darauf, dass es sich bei den Verbriefungen um ein weit verbreitetes Geschäftsmodell gehandelt habe. Auch branchenübliches Verhalten wird dann zu einem pflichtwidrigen Verhalten, wenn der die Existenz des Unternehmens gefährdende Charakter des Geschäftsmodells für den Täter erkennbar war ${ }^{104}$ und/oder von einem grundsätzlich anerkannten Geschäftsmodell exzessiv und/oder in ungewöhnlicher Form Gebrauch gemacht wird ${ }^{105}$. Dass die zum Tatzeitpunkt aktuellen Richtlinien des Basler Ausschusses für Bankenaufsicht (üblicherweise als Eigenkapitalvereinbarung oder Basel I bezeichnet) eine Eigenkapitalunterlegung bei der Auslagerung von Risiken in Zweckgesellschaften nicht zwingend vorgeschrieben haben, ist ebenfalls kein Argument das der Einstufung dieses Verhaltens als pflichtwidrig zwingend entgegensteht. Abgesehen davon, dass es sich bei diesen Richtlinien um keine für die Strafgerichte bei der Auslegung des Merkmals der Pflichtwidrigkeit verbindlichen Vorgaben handelt ${ }^{106}$, würde auch die Einhaltung verbindlicher Aufsichtsstandards die Einstufung als Pflichtwidrigkeit jedenfalls dann nicht ausschließen, wenn die Nichtinkaufnahme einer Existenzgefährdung zu den ausdrücklich oder konkludent vereinbarten Grundlagen des (Grund)Rechtsverhältnisses gehört ${ }^{107}$. Dies gilt umso mehr dann, wenn die Täter - was vorliegend der Fall gewesen sein soll108 - bewusst Lücken der aufsichtsrechtlich relevanten Regelungen suchen und ausnutzen.

Im Sinne eines Zwischenfazits bleibt festzuhalten: Es geht nicht an, jedes im

101 Zur Unzulässigkeit der Verschleifung der Tatbestandsmerkmale der Pflichtwidrigkeit und des Schadens vgl. BVerfGE 126, 170, 198 und 211; Deiters, in: Kempf/Lüderssen/Volk (Anm. 1), S. 133 f.; Krüger, NStZ 2011, 369, 375; Ransiek/Hüls, ZGR 2009, 157, 166 f.; Saliger, ZStW 112 (2000), S. 563, 610 f.; ders., HRRS 2006, 10, 14; Volk, Festschrift für Hamm, 2008, S. 803, 805 ff.

$102 \mathrm{Vgl}$. auch bereits Bosch/Lange, JZ 2009, 225, 228.

103 So auch Bittmann, NStZ 2011, 361, 366; Lutter, ZIP 2009, 197, 199; Park/Rütters, StV 2011, 434, 438; Schünemann, in: Schünemann (Anm. 1), S. 90 f.; Spindler, ZSG 2010, 281, 284; vgl. aber auch Brüning/Samson, ZIP 2009, 1089, 1092; Trüg, in: Kempf/Lüderssen/Volk (Anm. 1), S. 298.

104 Vgl. Fleischer NJW 2010, 1504, 1506; Schäfer/Zeller, BB 2009, 1706, 1710 f. (bezogen auf die zivilrechtliche Verantwortlichkeit).

105 Schröder, NJW 2010, 1169, 1172: Es sei nicht zu beanstanden, wenn von Fristentransformation mittels Zweckgesellschaften maßvoll Gebrauch gemacht wird.

106 Auch für die Strafgerichte verbindlich sind die im Rahmen der Selbstregulierung gesetzten Normen nur dann, wenn es sich um eine Normsetzung im Rahmen der staatlich gelenkten Selbstregulierung handelt; vgl. Woblers (Anm. 38), S. 305 ff.

107 Vgl. hierzu Schröder, Handbuch (Anm. 1), Rdn. 1170 ff.; ders., NJW 2010, 1169, 1171: Wenn aufsichtsrechtlich gesehen freie Hand bezüglich Eigenkapitalunterlegung besteht, werden Grenzen durch das Gebot der Existenzsicherung errichtet.

108 Vgl. Schröder, Handbuch (Anm. 1), Rdn. 1196. 
Zusammenhang mit Asset Backed Securities stehende Verhalten per se als pflichtwidrig einzustufen. Tatsächlich sind weder Verbriefungen ${ }^{109}$ noch Fristentransformationen ${ }^{110}$ per se illegitim und damit auch nicht per se pflichtwidrig. Es kann aber Fälle geben, in denen dies der Fall ist, weil Risiken eingegangen wurden, die nach dem konkret in Frage stehenden Grundverhältnis nicht eingegangen werden durften ${ }^{111}$. Bezogen auf die Situation in Deutschland scheint dies am ehesten bei den Landesbanken der Fall zu sein ${ }^{112}$.

\section{Die Schädigung des betreuten Vermögens}

Ist die Pflichtwidrigkeit gegeben, stellt sich weiter das Problem des Vermögensschadens. Die Frage, wann von einem Vermögensschaden bzw. - in der Diktion des deutschen und des österreichischen Untreuestrafrechts - von einem Vermögensnachteil ${ }^{113}$ gesprochen werden kann, die in den letzten Jahren in Deutschland zu einem der wohl meistdiskutierten Probleme des Vermögensstrafrechts avanciert ist, sollte allerdings im vorliegenden Zusammenhang jedenfalls dann keine unlösbaren Probleme aufwerfen, wenn man sich an die Vorgaben hält, deren Berücksichtigung nun auch das BVerfG als verfassungsrechtlich geboten anerkannt hat ${ }^{114}$.

Tatsächlich wird man die konkrete Gefährdung als schadensgleichen Nachteil einstufen können, wenn man den Gefährdungsschaden auf die Fallgestaltungen beschränkt, in denen aufgrund des in Frage stehenden Verhaltens des Täters ein aktueller/gegenwärtiger Minderwert und damit ein „echter“ Schaden gegeben ist $^{115}$. Die Frage ist, ab wann eine Gefährdung ein aktueller und nicht mehr

109 Hellwig (Anm. 1), E 16 ff.; Höfling, Finanzmarktregulierung - Welche Regelungen empfehlen sich für den deutschen und europäischen Finanzsektor?, Gutachten F zum 68. Deutschen Juristentag, 2010, F 54; Park/Rütters, StV 2011, 434, 437; Schröder, Handbuch (Anm. 1), Rdn. 1167; ders., NJW 2010, 1169, 1169/1170.

110 Schröder, Handbuch (Anm. 1), Rdn. 1169; ders., NJW 2010, 1169, 1171.

111 Park/Rütters, StV 2011, 434, 438.

112 Vgl. Lutter, BB 2009, 786 ff.; Schünemann, in: Schünemann (Anm. 1), S. 89 sowie - insbesondere zum Fall HSH-Nordbank - Strate, HRRS 2009, 441 f.; ders., Bucerius Law Journal 2009, $78 \mathrm{ff}$; zu diesen und weiteren, laufenden und abgeschlossenen Ermittlungsverfahren vgl. den Überblick bei Jahn, JZ 2011, 340, 343 f.

113 Zur Identität von Vermögensnachteil und Vermögensschaden vgl. BVerfG NJW 2009, 2370, 2371 mit Bespr. Fischer StV 2010, 95, 97; Lackner/Kübl, StGB, 27. Aufl. 2011, \266 Rdn. 17; Schünemann, in: LK (Anm. 47), \$266 Rdn. 132; Dierlamm (Anm. 47), \$266 Rdn. 177; Perron (Anm. 95), \266 Rdn. 39; Hoyer (Anm. 47), \266 Rdn. 93; Saliger (Anm. 47), \266 Rdn. 53; Beulke, Festschrift für Eisenberg, 2009, S. 258; Brüning/Samson ZIP 2009, 1089, 1091 sowie für das österreichische Recht-Schwaighofer/Venier, in: Bertel/Schwaighofer, Österreichisches Strafrecht, Besonderer Teil I, 11. Aufl., Wien 2010, S. 278 f.; kritisch gegenüber dieser Gleichsetzung Safferling, NStZ 2011, 376, 378.

114 Vgl. aber auch die dezidiert kritische Besprechung von Krüger, NStZ 2011, 369, 374 f. sowie Schünemann, StraFo 2010, 477, $480 \mathrm{ff}$.

115 BVerfGE 126, 170, 221 ff.; vgl. auch bereits BVerfG NJW 2009, 2370, 2372 mit Bespr. Fischer StV 2010, 95, 97 f.; BGHSt. 52, 323, 336 ff.; 53, 199, 202 ff.; 55, 266, 282 ff.; BGH NStZ 2008, 457; Nack, StraFo 2008, 277, 278 ff.; Saliger, Festschrift für Samson, 2010, S. 469 f.; zum Mei- 
nur ein potenzieller Schaden ist. Vollkommen zu Recht hat Saliger ausgeführt: die „Hauptschwierigkeit bei der schadensgleichen Vermögensgefahr besteht seit jeher darin, die schon schadensgleichen Verlustwahrscheinlichkeiten von den noch nicht schadensgleichen zu unterscheiden “ 116.

Die insoweit relevanten Abgrenzungsmaßstäbe waren in der deutschen Diskussion bisher hoch umstritten ${ }^{117}$. Nachdem nun auch das BVerfG diesen Ansatz unter verfassungsrechtlichen Gesichtspunkten als geboten anerkannt hat, dürfte es sich anbieten, im Anschluss an die in der Schweiz ganz herrschende Meinung ${ }^{118}$ darauf abzustellen, ob ein aktueller Wertberichtigungs- bzw. Abschreibungsbedarf gegeben ist ${ }^{119}$. Dies hat zur Folge, dass der Abschreibungsbedarf nicht nur vermutet und/oder aus dem später eingetretenen realen Schaden auf die konkrete Gefährdung rückgeschlossen wird. Soweit dem Gericht die entsprechende Sachkunde fehlt, wird es sich bei der Ermittlung dieses Bedarfs der Hilfe eines Sachverständigen bedienen müssen ${ }^{120}$. Bei Unklarheiten in tatsächlicher Hinsicht ist - in Anwendung des Grundsatzes in dubio pro reo ${ }^{121}$ - von der für den Täter vorteilhafteren Variante auszugehen und - soweit Prognose- und Beurteilungsspielräume bestehen - ist im Wege der Schätzung der als sicher anzunehmende Mindestschaden zu ermitteln ${ }^{122}$. Konkret bedeutet dies, dass nur der evident vorhandene Abschreibungs- und Wertberichtigungsbedarf als

nungsstand zum Gefährdungsschaden im deutschen Schrifttum vgl. Schünemann, in: LK (Anm. 47), \$266 Rdn. 146; Kindhäuser (Anm. 48), \$266 Rdn.110; Perron (Anm. 95), \$266 Rdn. 45 ff.; Saliger (Anm.47), \$266 Rdn. 66 ff.; zum schweizerischen Recht vgl. BGE 121 IV 104, 107; 122 IV 279, 281; Niggli (Anm. 48), Art. 158 Rdn.114; Stratenwerth/Woblers (Anm. 30), Art. 158 Rdn. 5.

116 Saliger, Festschrift für Samson, 2010, S. 471. Für das deutsche Recht akzentuiert sich die Notwendigkeit, diese Frage zu klären, noch dadurch, dass die Erfassung abstrakter Vermögensgefährdungen die Straflosigkeit des Versuchs unterlaufen würde; vgl. hierzu BVerfG NJW 2009, 2370, 2372; Hoyer (Anm. 47), \$266 Rdn. 101; Beulke, Festschrift für Eisenberg, 2009, S.262; Rönnau ZStW 122 (2010), S.299, 305, 306.

117 Vgl. Dierlamm (Anm. 47), $\$ 266$ Rdn. 186 ff.; Beulke, Festschrift für Eisenberg, 2009, S. 262; Saliger ZStW 112 (2000), S. 563, 565 f. sowie S. $574 \mathrm{ff}$.

118 Vgl. BGE 122 IV 279, 281; 123 IV 17, 22; 129 IV 124, 125 f.; Donatsch, schwZStrR 114 (1996), S. 200, 217; ders., schwZStrR 120 (2002), S. 1, 22; ders. (Anm. 55), S. 280; Schubarth, Festschrift für Gauthier, Bern 1986, S.79; Stratenwerth/Woblers (Anm. 30), Art.158 Rdn. 5; Trechsel/ Crameri (Anm. 48), Art. 158 Rdn. 12.

119 BVerfGE 126, 170, 223 ff.; Nack, StraFo 2008, 277, 279; Schröder, Handbuch (Anm. 1), Rdn. 1192; ders., NJW 2010, 1169, 1174; Steinberg/Dinter, JR 2011, 224, 227; zur Orientierung am Bilanzrecht vgl. auch bereits Hefendebl, Vermögensgefährdung und Exspektanzen, 1994, S. $166 \mathrm{ff}$. und passim; ders., Festschrift für Samson, 2010, S. $301 \mathrm{ff}$.; kritisch zur Schadensberechnung unter Heranziehung bilanzrechtlicher Methoden Becker, HRRS 2009, 334, 338; Fischer, NStZ-Sonderheft für Miebach, 2009, 8, 12; ders., StV 2010, 95, 101; Wessing/ Krawczyk, NZG 2010, 1121, 1124; anderer Ansatz bei Kasiske, in: Schünemann (Anm. 1), S. 31 ff., der den Schaden aus der Unvertretbarkeit des eingegangenen Risikos ableiten will; a. A. auch Fischer, StV 2010, 95, 100 f.; ders., StraFo 2010, 329, 334: es sei am Gefährdungsschaden als besondere, normativ bestimmte Form eines Schadens festzuhalten.

120 BVerfGE 126, 170, 225 f.; Bittmann, NStZ 2011, 361; Jabn, JZ 2011, 340, 346; Safferling, NStZ 2011, 376, 378.

121 BVerfGE 126, 170, 211 f. sowie 225; BGHSt. 53, 199, 203; BGH NStZ 2008, 457.

122 BVerfGE 126, 170, 225 f.; BGHSt. 53, 199, 203; BGH NStZ 2008, 457. 
Schaden anerkannt werden kann ${ }^{123}$ und die Höhe des Schadens durch diesen im unmittelbaren Anschluss an die Tathandlung gegebenen Abschreibungs- und Wertberichtigungsbedarf begrenzt wird ${ }^{124}$. Die weitere Entwicklung ist dagegen irrelevant ${ }^{125}$, d. h. weder wird der Schaden dadurch größer, dass sich das Risiko später tatsächlich realisiert ${ }^{126}$, noch verschwindet der Schaden rückwirkend, wenn sich das Risiko aufgrund sonstiger glücklicher Umstände doch nicht realisiert $^{127}$.

Gegen das skizzierte Modell der Ermittlung des Gefährdungsschadens wird eingewandt, dass dieses den Strafgerichten zu viel Aufwand mache bzw. der notwendige Aufwand mit den vorhandenen personellen und sachlichen Ressourcen nicht zu bewältigen sei. So meint Fischer: „Die große Mehrzahl der Tatrichter wird die ,International Accounting Standards' der finanztechnischen Bewertung von Kreditrisiken nicht kennen, anwenden wollen oder können "128. Ausgehend von der Unbestimmtheit der Bilanzierungs- und Bewertungsregeln sei der Abschreibungs- bzw. Wertberichtigungsschaden im Übrigen nichts anderes als ein geschätzter Schaden, mit dem nichts anderes erreicht werde, als die Tür zu Absprachen noch weiter $\mathrm{zu}$ öffnen ${ }^{129}$.

Dem ist zunächst entgegen zu halten, dass die sachlich gebotene Ermittlung des konkreten Abschreibungs- bzw. Wertberichtigungsbedarfs nicht deshalb unterbleiben darf, weil sie mit praktischen Schwierigkeiten verbunden ist ${ }^{130}$ und/ oder auf den Widerwillen der Instanzrichter stößt. Die praktischen Schwierigkeiten dürften sich im Übrigen im Rahmen des Vertretbaren halten, wenn man berücksichtigt, dass es darum geht, den evident vorhandenen Abschreibungsund Wertberichtigungsbedarf als Basis für eine Bestimmung des Mindestschadens zu ermitteln.

Und wenn Nack daran festhalten will, dass die Strafgerichte das Vorliegen eines Schadens allein auf ein nicht näher spezifiziertes signifikantes Ausfallrisiko stützen können sollen und nur im Rahmen der Strafzumessung ein unter Berücksichtigung des Zweifelssatzes freihändig vorgenommener Abschlag erfolgen soll131, dann läuft dies letztlich darauf hinaus, einer moralistischen Schadens-

123 Becker, HRRS 2009, 334, 339 f.; Steinberg/Dinter, JR 2011, 224, 227.

124 Brüning/Samson, ZIP 2009, 1089, 1094; Jahn, JZ 2011, 340, 346; Nack, StraFo 2008, 277, 279 f.; Park/Rütters, StV 2011, 434, 439 f.; Schröder, Handbuch (Anm.1), Rdn. 1195. Relevant ist allein der objektive Abschreibungsbedarf; ob tatsächlich so bilanziert worden wäre, ist irrelevant; vgl. Bittmann, NStZ 2011, 361, 368.

125 BGHSt. 53, 199, 202.; Ransiek, WM 2010, 869, 872.

126 Park/Rütters, StV 2011, S. 434, 439.

127 Schröder, Handbuch (Anm. 1), Rdn. 1193; BGH NStZ 2008, 457; 2011, 403, 406; a. A. BGHSt. 51, 100, 121; BGH NStZ 2007, 704.

128 Fischer, StraFo 2010, 329, 334/335.

129 Vgl. Fischer, NStZ-Sonderheft für Miebach, 2009, 8, 12 und 15; ders., StV 2010, 95, 101; ders., StraFo 2010, 329, 334 f.; Nack StraFo 2008, 277, 280.

130 BVerfGE 126, 170, $211 \mathrm{f}$.

131 Nack, StraFo 2008, 277, 280. 
zurechnung Tür und Tor zu öffnen ${ }^{132}$. Dass es sich hierbei nicht nur um eine abstrakte, sondern um eine höchst konkrete Gefahr handelt, belegt die Rechtsprechung des 1. Strafsenats des BGH, nach der bei einem Schneeballsystem auch dann ein Schaden angenommen wird, wenn der Täter realistischerweise mit einer Rückzahlung rechnen konnte ${ }^{133}$. Auch wenn das Mitmachen bei einem Schneeballsystem moralisch verwerflich erscheint, weil zwingenderweise am Ende (irgend)jemand geschädigt werden muss: Dass man dieses Verhalten auch dann als (vermögens-)strafrechtlich relevant einzustufen hat, wenn der Täter berechtigterweise davon ausgehen durfte, dass er das von ihm zu betreuende Vermögen nicht schädigen wird, wird man nicht ohne weiteres annehmen können.

\section{Die subjektive Tatseite}

Der Untreuetäter muss vorsätzlich handeln, wobei nach unbestrittener Auffassung auch bedingter Vorsatz ausreichend ist. Entscheidend ist, dass der Täter im Tatzeitpunkt zumindest die Möglichkeit erkannt hat, dass sein Verhalten als pflichtwidrig einzustufen ist und das von ihm zu betreuende Vermögen zumindest in der Form eines Gefährdungsschadens einen Nachteil erleidet. Wenn man mit der hier vertretenen Auffassung den durch den Abschreibungs- und Wertberichtigungsbedarf begrenzten Vermögensschaden anerkennt, gibt es keinen Grund, im Hinblick auf den Schädigungsvorsatz zu verlangen, dass der Täter die spätere Realisierung dieses Schadens als ernsthaft möglich eingestuft hat ${ }^{134}$. $\mathrm{Ob}$ der Täter sein Verhalten als pflichtwidrig und vermögensschädigend eingestuft hat - und ihm dies in einer den Anforderungen an eine strafrechtliche Verurteilung genügenden Art und Weise - nachgewiesen werden kann, ist eine Frage des Einzelfalls, wobei in der Literatur die Annahme eines vorsätzlichen Verhaltens wohl zutreffend jedenfalls für die Fälle bejaht wird, in denen es um die weitere Mitwirkung nach Publikwerden der Krise geht ${ }^{135}$.

Besondere Probleme ergeben sich dann, wenn der Täter im Tatzeitpunkt davon ausgegangen ist - bzw. ihm dies nicht als Schutzbehauptung widerlegt werden kann -, dass er sich pflichtgemäß verhalten habe. Ansatzpunkt zur Beant-

132 Kritisch hierzu bereits Schünemann, StraFo 2010, 477, 479.

133 BGHSt. 53, 199, 204 f.; kritisch hierzu Saliger, Festschrift für Samson, 2010, S. 467 f. sowie $474 \mathrm{ff}$.

134 So aber BGHSt. 51, 100, 121; BGH NStZ 2007, 704 mit Bespr. Schlösser, NStZ 2008, 397; Deiters, in: Kempf/Lüderssen/Volk (Anm. 1), S. 135; Fischer, NStZ-Sonderheft für Miebach, 2009, 8, 13 f.; kritisch hierzu Kindhäuser (Anm. 48), \$266 Rdn. 123; Beulke, Festschrift für Eisenberg, 2009, S. 263 f.; wie hier dagegen BGHSt. 47, 148, 157; 48, 331, 346; 53, 199, 204; BGH NStZ 2008, 457; 2011, 403, 406; Bosch/Lange, JZ 2009, 225, 236 f.; Hefendehl, Festschrift für Samson, 2010, S. 304 f.; Hillenkamp, Festschrift für Maiwald, 2010, S. 338 ff.; Nack, StraFo 2008, 277, 281; Steinberg/Dinter, JR 2011, 224, 227; vgl. auch Ransiek/Hüls, ZGR 2009, 157, 167 ff.; Hoyer (Anm.47), \$266 Rdn. 105; im Ergebnis übereinstimmend Kasiske, in: Schünemann (Anm. 1), S. $36 \mathrm{f}$.

135 Vgl. Park/Rütters, StV 2011, 434, 440; Gallandi, wistra 2009, 41, 46; Schröder, Handbuch (Anm. 1), Rdn. 1200; vgl. ders. NJW 2010, 1169, 1174. 
wortung dieser Frage ist die umstrittene Einordung des Irrtums über die Pflichtwidrigkeit des Verhaltens ${ }^{136}$. Stuft man die irrtümliche Annahme der Pflichtgemäßheit des Verhaltens als Verbotsirrtum ein, was insbesondere dann konsequent ist, wenn man die Pflichtwidrigkeit als ein gesamttatbewertendes Merkmal einstuft ${ }^{137}$, dann ist die Sache relativ klar: Da dieser Irrtum - jedenfalls gemessen an den üblicherweise angelegten Maßstäben ${ }^{138}$ - wohl vermeidbar war, kommt es allenfalls zu einer Strafmilderung. Sieht man in der Pflichtwidrigkeit dagegen ein normatives Tatbestandsmerkmal ${ }^{139}$ wird man den Irrtum wohl als Tatbestandsirrtum einstufen müssen ${ }^{140}$, was dann $\mathrm{zu}$ einer differenzierten Betrachtungsweise zwingt: Hat der entweder rechtlich selbst versierte oder doch zumindest beratene Täter erkannt, dass sein Verhalten als pflichtwidrig eingestuft werden könnte, wird man ihm einen bedingten Vorsatz bezogen auf die Pflichtwidrigkeit attestieren können. Ist der Täter dagegen - gegebenenfalls auch blauäugig - davon ausgegangen, dass er sich zweifelsfrei pflichtgemäß verhalte, wird man ihm einen Tatbestandsirrtum zubilligen müssen ${ }^{141}$. Wirklich beunruhigende Strafbarkeitslücken dürften aber auch unter Zugrundelegung dieses Ansatzes letztlich nicht auftreten, weil in den Fällen, in denen bei restriktiver Interpretation des objektiven Tatbestands die Pflichtwidrigkeit des in Frage stehenden Verhaltens objektiv gegeben ist, die Einlassung, man habe diese Wertung nicht einmal laienhaft nachvollzogen, regelmäßig als Schutzbehauptung erkennbar und damit widerlegbar sein wird. Und in den Fällen, in denen dies nicht der Fall ist, muss man sich damit abfinden, dass das geltende Recht - aus guten Gründen ${ }^{142}$ auf die Bestrafung fahrlässiger Untreue verzichtet. Diese Wertung des Gesetzgebers darf der Rechtsanwender dann aber auch nicht dadurch konterkarieren, dass er auf das Instrument von Verdachtsbestrafungen zurückgreift ${ }^{143}$.

\section{Abschließende Würdigung}

Das Strafrecht - und damit auch der Untreuestraftatbestand - ist ein per se ungeeignetes Instrument, wenn es darum geht, zukünftige Finanzkrisen prospek-

136 Vgl. hierzu auch bereits Deiters, in: Kempf/Lüderssen/Volk (Anm. 1), S. $137 \mathrm{f}$.

137 So Kindhäuser (Anm. 48) Art. 266 Rdn. 122; Kasiske, in: Schünemann (Anm. 1), S. 35; Schünemann, in: Schünemann (Anm. 1), S. 97; ders., Organuntreue (Anm. 47), S. 66; Schünemann, in: LK (Anm. 47), $\$ 266$ Rdn. 153.

138 Vgl. nur Lackner/Kühl (Anm.113), $\$ 17$ Rdn. 7; Neumann, in: Nomos Kommentar zum Strafgesetzbuch, $\$ 17$ Rdn. $58 \mathrm{ff}$.

139 BVerfGE 126, 170, 204 mit zust. Bespr. Saliger, NJW 2010, 3195, 3196; BGHSt. 55, 288, 300; Tiedemann, Festschrift für Weber, 2004, S. 322.

140 Perron (Anm. 95), \$266 Rdn. 49; Dierlamm (Anm. 47), \$266 Rdn. 239; Bittmann, NStZ 2011, 361, 366; Jakobs, NStZ 2005, 276, 277.

141 So auch Beulke, Festschrift für Eisenberg, 2009, S. 265.

142 Vgl. auch - zum Vorschlag, mit Blick auf die Beweisschwierigkeiten de lege ferenda auch die fahrlässige Untreue unter Strafe zu stellen - Otto, Festschrift für Krey, 2010, S. 402 f.

143 Vgl. Niggli (Anm. 48), Art. 158 Rdn.116: „Auch die grobfahrlässige Pflichtverletzung bleibt straflos." 
tiv verhindern zu wollen. Das Untreuestrafrecht ist aber ein Instrument, das im Rahmen der Aufarbeitung der eingetretenen Finanzkrise zur Anwendung kommen kann und - soweit die Strafbarkeitsvoraussetzungen im konkreten Einzelfall gegeben $\operatorname{sind}^{144}$ - auch zur Anwendung kommen muss. Wann dies der Fall ist, wird die Rechtsprechung in den zur Anklage kommenden Fällen zu entscheiden haben. Dass der Verdacht der Untreue im Ergebnis seltener begründet sein dürfte, als es die gegenwärtige, recht aufgeregte (fach-)öffentliche Debatte vermuten lässt ${ }^{145}$, wird man ohne weiteres annehmen können. Dass es nicht ausgeschlossen sei, dass der Verdacht in keinem einzigen Fall in ausreichendem Maße erhärtet werden kann ${ }^{146}$, ist eine Aussage, die natürlich einerseits für sich gesehen nicht falsch ist, die aber andererseits durch die Praxis auch schnell widerlegt werden kann - und mit einiger Wahrscheinlichkeit auch widerlegt werden wird.

144 Die Notwendigkeit der gesonderten Bewertung jedes einzelnen Falles betont zu Recht auch Schröder, Handbuch (Anm. 1), Rdn. 1201.

145 Deiters, in: Kempf/Lüderssen/Volk (Anm. 1), S. 138.

146 Bittmann, NStZ 2011, 361, 367. 